\title{
$1 \quad$ Nigrostriatal Dopamine Signals Sequence-Specific Action-Outcome Prediction Errors
}

2 Nick G. Hollon ${ }^{1}$, Elora W. Williams ${ }^{1}$, Christopher D. Howard ${ }^{1,2}$, Hao Li ${ }^{1}$, Tavish I. Traut ${ }^{1}$, and Xin

$3 \operatorname{Jin}^{1 *}$

$4{ }^{1}$ Molecular Neurobiology Laboratory, The Salk Institute for Biological Studies, La Jolla, CA

592037, USA.

$6 \quad{ }^{2}$ Current address: Neuroscience Department, Oberlin College, Oberlin, OH 44074, USA.

7 * Correspondence: xjin@salk.edu

\section{ABSTRACT}

9 Dopamine has been suggested to encode cue-reward prediction errors during Pavlovian

10 conditioning. While this theory has been widely applied to reinforcement learning concerning

11 instrumental actions, whether dopamine represents action-outcome prediction errors and how it

12 controls sequential behavior remain largely unknown. Here, by training mice to perform

13 optogenetic intracranial self-stimulation, we examined how self-initiated goal-directed behavior

14 influences nigrostriatal dopamine transmission during single as well as sequential instrumental

15 actions. We found that dopamine release evoked by direct optogenetic stimulation was

16 dramatically reduced when delivered as the consequence of the animal's own action, relative to

17 non-contingent passive stimulation. This action-induced dopamine suppression was specific to

18 the reinforced action, temporally restricted to counteract the expected outcome, and exhibited

19 sequence-selectivity consistent with hierarchical control of sequential behavior. Together these

20 findings demonstrate that nigrostriatal dopamine signals sequence-specific prediction errors in

21 action-outcome associations, with fundamental implications for reinforcement learning and

22 instrumental behavior in health and disease. 


\section{INTRODUCTION}

25 Our brains constantly generate predictions about the world around us (Rao and Ballard, 1999;

26 Keller and Mrsic-Flogel, 2018), particularly regarding the expected consequences of

27 environmental cues or our own actions (Wolpert et al., 1995; Crapse and Sommer, 2008;

28 Schneider et al., 2018; Wurtz, 2018). Indeed, the effects of such expectations have long been

29 recognized when examining the phasic activity of midbrain dopamine neurons following reward-

30 predictive stimuli during Pavlovian conditioning (Fiorillo et al., 2003). Many dopamine neurons

31 signal errors in cued reward prediction (Houk et al., 1995; Montague et al., 1996; Schultz et al.,

32 1997; Cohen et al., 2012; Eshel et al., 2015, 2016; Engelhard et al., 2019), i.e., any change in

33 expectation of future reward or difference between actual versus expected reward predicted by

34 the cues (Sutton and Barto, 2018). These dopaminergic prediction errors are thought to convey

35 a teaching signal that is critical for multiple forms of associative learning across the

36 corticostriatal topography (Yin et al., 2008; Balleine, 2019), spanning both classical Pavlovian

37 stimulus-outcome conditioning (Flagel, et al., 2011; Steinberg et al., 2013; Chang et al., 2016;

38 Saunders et al., 2018; Maes et al., 2020) and the formation of stimulus-response habits

39 (Knowlton et al., 1996; Matsumoto et al., 1999; Faure et al., 2005; Belin and Everitt, 2008;

40 Wang et al., 2011; Kim et al., 2015).

42 However, the vast majority of previous studies examining dopamine responses primarily have 43 used discrete reward-predictive stimuli (Schultz et al., 1997; Fiorillo et al., 2003; Morris, et al.,

44 2004; Roesch et al., 2007; Flagel, et al., 2011; Hollon et al., 2014; Cohen et al., 2012; Eshel et

45 al., 2015, 2016; Matsumoto and Hikosaka, 2009; Parker et al., 2016; Coddington and Dudman,

46 2018; Engelhard et al., 2019), whether Pavlovian conditioned stimuli, for which no action is

47 required to earn reward, or explicit discriminative stimuli that essentially instruct an animal how

48 and when to respond to earn reward. Although such explicit cues can exert powerful influences

49 over behavior, far less is known regarding dopamine's potential roles in and interactions with

50 goal-directed behavior that is self-initiated, self-paced, and guided by instrumental action-

51 outcome associations.

53 Parallel work also has sparked a renewed focus on dopamine in movement control (Klaus et al.,

54 2019; Coddington and Dudman, 2019). Across multiple recording modalities, several recent

55 studies have reported prominent changes in dopamine activity at the initiation of spontaneous

56 movements ranging from operant action sequences (Jin and Costa 2010; Wassum et al., 2012; 
57 Collins et al., 2016; da Silva et al. 2018) to locomotion and brief postural adjustments (Barter et

58 al., 2015; Dodson et al., 2016; Howe and Dombeck, 2016; da Silva et al. 2018; Coddington and

59 Dudman, 2018). However, the extent to which such movements were directed toward any

60 particular goal in the latter studies is unclear, and how self-initiated goal-directed actions

61 influence nigrostriatal dopamine release is largely unknown. Therefore, despite longstanding

62 implication in voluntary movement, motivation, and reinforcement learning, the precise role of

63 dopamine in instrumental action remains poorly understood. Here, we examined how goal-

64 directed action and learned action sequences influence the nigrostriatal dopamine response to

65 the consequence of these actions, in behavioral contexts with minimal overt changes in the

66 animal's external environment.

\section{RESULTS}

70 Suppression of Optogenetically Stimulated Nigrostriatal Dopamine by Goal-Directed Action

72 Mice expressing channelrhodopsin-2 selectively in their dopamine neurons (See Methods;

73 Zhuang et al., 2005; Madisen et al., 2012) were implanted with a fiber optic over the substantia

74 nigra pars compacta (SNc) for optogenetic stimulation (Sparta et al., 2011) and a carbon-fiber

75 microelectrode (Clark, Sandberg et al., 2010) in the ipsilateral dorsal striatum to record

76 nigrostriatal dopamine transmission using fast-scan cyclic voltammetry (FSCV; Figure 1A,

77 Figure 1-figure supplement $1 \mathrm{~A}-\mathrm{F})$. The mice were trained in a free-operant optogenetic

78 intracranial self-stimulation (opto-ICSS) task (Figure 1B), in which they learned to press a

79 continuously reinforced "Active" lever to optogenetically stimulate their own dopamine neurons

$80 \quad(50 \mathrm{~Hz}$ for $1 \mathrm{~s})$ and rarely pressed the non-reinforced "Inactive" lever yielding no outcome

81 (Figure 1C). Therefore, consistent with other recent reports (Rossi et al., 2013; Ilango et al.,

82 2014; Keiflin et al., 2018; Saunders et al., 2018), selective stimulation of SNc dopamine neurons

83 is sufficient to reinforce novel actions.

85 To examine the extent to which this behavior is indeed goal-directed, a subset of mice

86 underwent a contingency degradation test (Witten, et al., 2011; Koralek, et al., 2012; Clancy et

87 al., 2014; Neely et al., 2018). During this test phase, stimulation was decoupled from the lever-

88 pressing action and instead delivered non-contingently at a rate yoked to that animal's own

89 stimulation rate from the preceding self-stimulation phase (Methods). The mice significantly 
90 reduced their performance rate (Figure 1D, Figure 1-figure supplement $1 \mathrm{G}-\mathrm{H}$ ), indicating that

91 they readily learned that their action was no longer required to earn stimulation. This

92 demonstrates that nigrostriatal dopamine neuron self-stimulation under this simple fixed-ratio

93 schedule of continuous reinforcement (CRF) is sensitive to changes in the action-outcome

94 contingency, which is an established operational hallmark of goal-directed behavior (Yin et al.,

95 2008; Balleine, 2019).

97 To investigate whether goal-directed action affects the nigrostriatal dopamine response to the

98 consequence of that action, we used FSCV to record subsecond dopamine transmission in

99 behaving mice during sessions that included two phases: In the Self-Stimulation phase, as in

100 prior opto-ICSS training, mice earned optogenetic stimulation for each Active lever press (Figure

101 1B). In the subsequent Passive Playback phase, the levers were retracted, and the mice

102 received non-contingent stimulations, with timestamps identical to the stimulations that each

103 individual had self-administered in its Self-Stimulation phase. Thus, in this entirely within-subject

104 design, we recorded at the same striatal location with the same chronically implanted electrode,

105 with each animal yoked to its own performance, receiving the same temporal sequence of

106 stimulations across both phases of the session, delivered to the same site within the SNc using

107 identical optogenetic stimulation parameters to directly depolarize these nigrostriatal dopamine

108 neurons (Figure $1 \mathrm{~A}$ and $1 \mathrm{E}-\mathrm{F}$ ).

110 We observed a remarkably robust difference between the amplitude of Self-Stimulated

111 dopamine release and the significantly greater amplitude evoked by the non-contingent Passive

112 Playback stimulation (Figure 1F-I). All individual mice (9/9, 100\%) exhibited less dopamine

113 release when evoked as the consequence of their own action; this difference was significant at

114 the individual level in 7 of 9 mice $(P s<0.0001)$ and was a trend in the same direction for the

115 remaining 2 mice ( $P S=0.0623$ and 0.0825 ). Although the free-operant opto-ICSS task was

116 designed to minimize discrete external cues, it nevertheless is possible that the offset of a

117 previous stimulation essentially could serve as a stimulus that might elicit the next lever-

118 pressing response. However, when we isolated the initiation of lever-pressing bouts using an

119 inter-stimulation interval (ISI) criterion of at least $10 \mathrm{~s}$ since the previous stimulation, this subset

120 of stimulations still showed a significant difference between Self-Stimulated and non-contingent

121 Playback-evoked dopamine release (Figure 1-figure supplement 1I-K). This finding further

122 indicates that optogenetically evoked dopamine release is lower when it is the outcome of self-

123 initiated, goal-directed actions. 


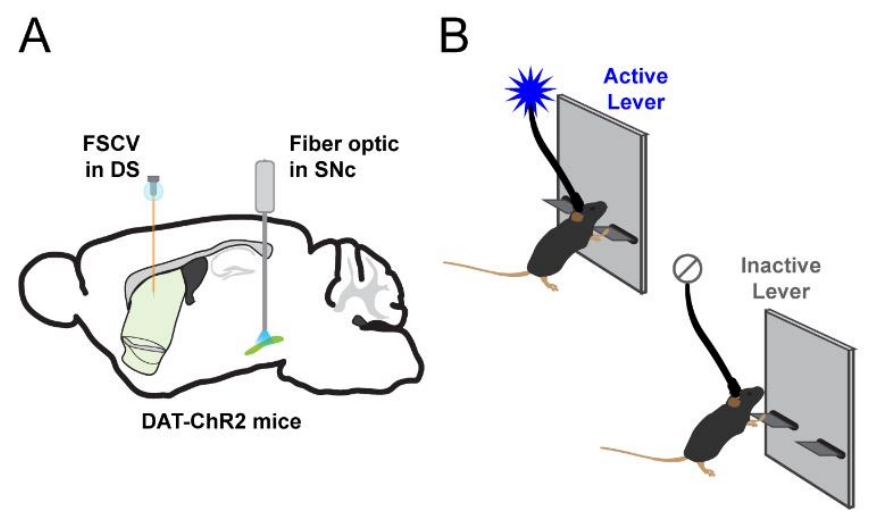

$\mathrm{E}$

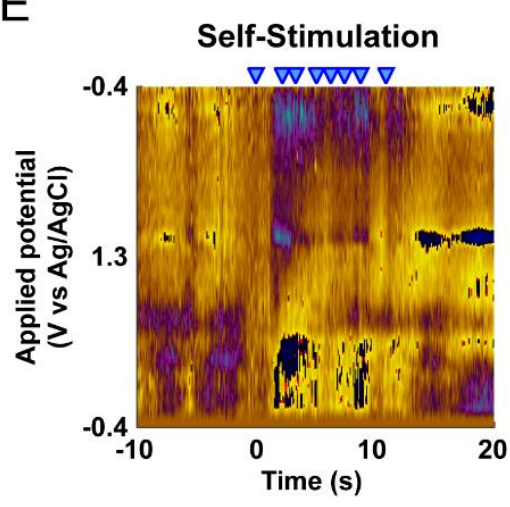

G

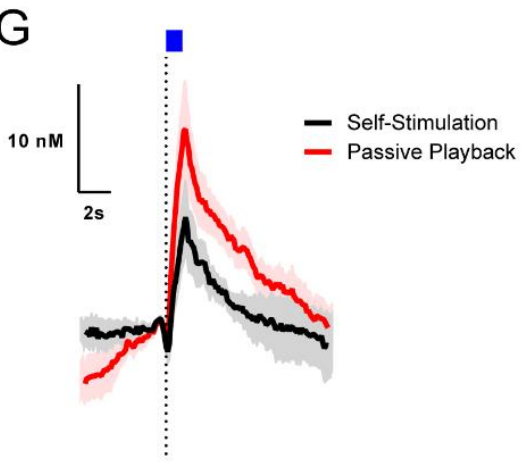

C

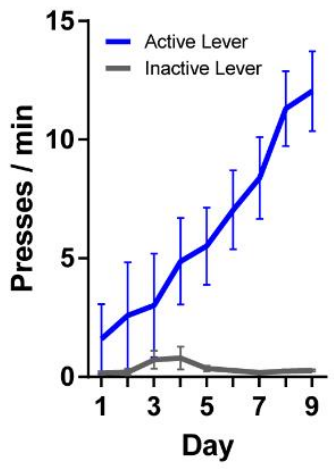

D

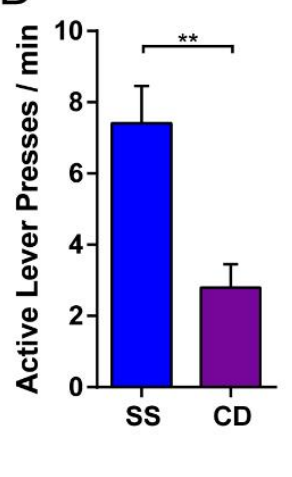

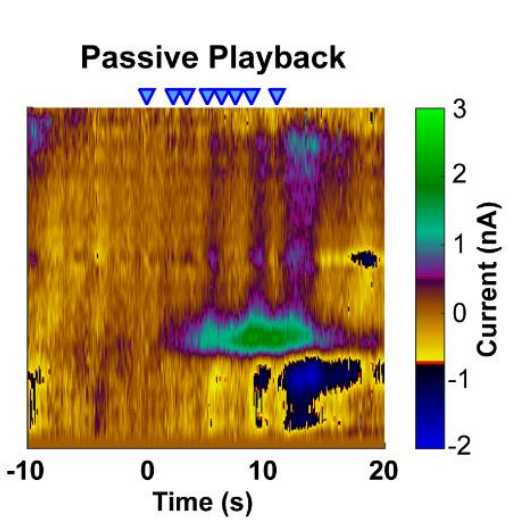

F
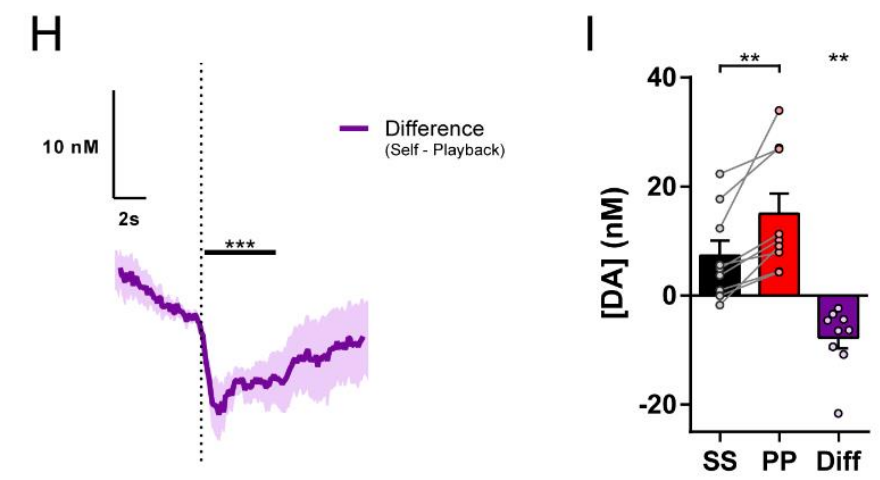

Figure 1. Goal-Directed Action Suppresses Nigrostriatal Dopamine Release During OptoICSS. (A) Schematic of experimental preparation for optogenetic stimulation of SNc dopamine neurons and FSCV recording in dorsal striatum. (B) Opto-ICSS behavioral task schematic. (C) Acquisition of opto-ICSS ( $n=9$ mice; two-way repeated-measures ANOVA: main effect of Lever, $F_{1,8}=16.84, P=0.0034$; main effect of Day, $F_{8,64}=9.314, P<0.0001$; Lever by Day interaction, $F_{8,64}=10.63, P<0.0001$; Active Lever significantly greater than Inactive Lever for Days 4-9, $P S \leq 0.008$ ). (D) Contingency degradation test: $30 \mathrm{~min}$ of opto-ICSS followed by 30 min contingency degradation test phase $\left(n=6\right.$ mice; paired t test, $\left.t_{5}=5.441, P=0.0028\right)$. (E) Representative voltammetric pseudocolor plots from a bout of stimulations (blue triangles) during the Self-Stimulation phase (left) and the matched stimulations from the Passive Playback phase (right). (F) Dopamine responses to the series of stimulations in each session phase from the example in (E). Inset: cyclic voltammograms. (G) Mean change in dopamine concentration evoked by Self-Stimulation and Passive Playback stimulations ( $n=9$ mice). (H) Difference 
138 trace: Self-Stimulation minus Passive Playback, from traces in (G). Black bar indicates poststimulation time points with significant difference vs. 0 (permutation test, $P=0.0001$ ). (I) Mean change in dopamine concentration (Self-Stimulation vs. Passive Playback paired t test, $t_{8}=$ 3.923, $P=0.0044$; equivalently, Difference vs. 0 one-sample t test: $t_{8}=3.923, P=0.0044$ ). SS, Self-Stimulation; CD, Contingency Degradation; PP, Passive Playback; Diff, Difference (Self minus Playback). All error bars are SEM, same for below unless stated otherwise. See also Figure 1-figure supplement 1.

\section{Nigrostriatal Dopamine Signals Action-Outcome Prediction Errors}

147 The reward prediction error theory implies decreased dopamine responses to expected versus

148 unexpected outcomes (Houk et al., 1995; Montague et al., 1996; Schultz et al., 1997; Cohen et

149 al., 2012; Eshel et al., 2016; Sutton and Barto, 2018). Nevertheless, the relative difference we

150 observed does not alone resolve whether dopamine release is in fact inhibited by the animal's

151 action. To address this question, we recorded additional sessions in which a random $20 \%$ of

152 Active lever presses did not yield stimulation, instead causing a 5-s timeout period during which

153 no further stimulation could be earned (Figure 2A). During these Omission Probes, there was a

154 clear dip in dopamine below baseline levels (Figure 2B-C), consistent with a neurochemical

155 instantiation of a negative prediction error (Hart et al., 2014). Indeed, the timecourse for this

156 Omission Probe dip was remarkably similar to the digital subtraction ("Difference Trace") of the

157 Self-Stimulated dopamine response minus the Passive Playback response (Figure 1H; overlaid

158 in Figure 2D). This Omission Probe dip was not merely an artifact of FSCV background

159 subtraction (Hamid et al., 2016), where reuptake during the stimulation-free timeout period

160 might follow an elevated baseline from several preceding stimulations. Rather, a significant dip

161 below baseline was still prominent for the subset of Omission Probes with a minimum latency of

162 at least $5 \mathrm{~s}$ since the previous stimulation, whereas no such decrease was detected at the

163 equivalent time points from the Playback phase (Figure 2-figure supplement 1A-B).

164 Furthermore, additional lever presses during an ongoing stimulation augmented the suppression

165 of Self-Stimulated dopamine release, and similarly, additional presses during an Omission

166 Probe timeout period prolonged the duration of the dip below baseline (Figure 2-figure

167 supplement 1C-F). In vivo extracellular electrophysiological recording further revealed reduced

168 somatic firing in optogenetically-identified SNc dopamine neurons in response to action-evoked

169 optogenetic Self-Stimulation relative to non-contingent Passive Playback stimulation (Figure 2-

170 figure supplement 1G-L). Collectively, these results demonstrate that the action indeed causes

171 inhibition of dopamine transmission.

172 
173 It recently has been reported that some dopamine neurons transiently reduce their firing rate

174 during certain types of spontaneous movement (Dodson et al., 2016; da Silva et al. 2018;

175 Coddington and Dudman, 2018). We therefore considered the possibility that the action-induced

176 suppression observed in our recordings may be a generalized inhibition following any lever

177 pressing action, regardless of whether that action is associated with a particular reinforcing

178 outcome. However, we found no such inhibition in the instances when the animal pressed the

179 Inactive lever, which had never been reinforced throughout training (Figure 2D-E), indicating

180 that the action-induced inhibition of dopamine release is specific to the typically reinforced

181 action and conveys a bona fide prediction-error signal. Furthermore, because the sensory

182 feedback from pressing the Active and Inactive levers is rather similar to the animals, these

183 results confirmed that the dopamine inhibition results from the expectations associated with

184 specific self-initiated, goal-directed action but not simply a conditioned sensory cue.

186 To examine the temporal specificity of this action-induced suppression, we recorded Delay

187 Probe sessions in which $20 \%$ of Active lever presses instead resulted in stimulation that was

188 delayed by $5 \mathrm{~s}$ (Figure $2 \mathrm{~F}$ ). The initial $5 \mathrm{~s}$ of this delay period was equivalent to the timeout

189 period of the Omission Probes, and we again observed a dip in dopamine below baseline

190 (Figure 2G-H). When the probe stimulation was finally delivered at the end of the delay period,

191 there now was a high amplitude of dopamine release that did not differ from the corresponding

192 Playback stimulations (Figure 2I-J). Because these Delay Probes were randomly interleaved

193 throughout the Self-Stimulation phase, this indicates that there is not a global suppression of

194 dopamine neuron excitability throughout the whole context of the Self-Stimulation phase.

195 Rather, this action-induced inhibition is precisely timed to counteract the expected consequence

196 of that action, namely the immediate stimulation that is its typical outcome.

198 We further determined the nature of this action-induced inhibition in Magnitude Probe sessions,

199 where $20 \%$ of Active lever presses yielded $5 \mathrm{~s}$ of stimulation rather than the standard 1-s

200 stimulation used throughout training (Figure 2K). These increased Magnitude Probes indeed

201 evoked much greater dopamine release, as expected for longer-duration stimulation (Figure 2L-

202 M). Closer examination of the time course of these dopamine responses also revealed a

203 transient suppression during the self-stimulated Magnitude Probes that was restricted to the first

204 second or so following stimulation onset, but no longer differed from Probe Playback by the end

205 of the 5-s probe stimulation. This brief inhibition also was borne out by the transient dips and

206 similar overall time courses in the Difference traces for both the Magnitude Probes and the 
standard 1-s stimulations, comparing each type of Self-Stimulation to their respective Playback stimulations (Figure $2 \mathrm{~N}-\mathrm{O}$ ). This again highlights the timing and duration specificity of the actioninduced suppression, and suggests that there is not a global inhibition of dopamine throughout the Self-Stimulation context. Together, these data suggest that nigrostriatal dopamine can encode a reward prediction error signal for individual goal-directed action and its expected

212 outcome.

A

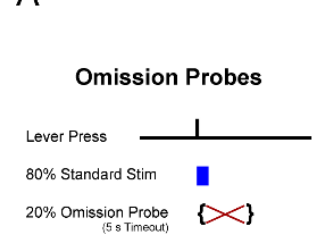

B

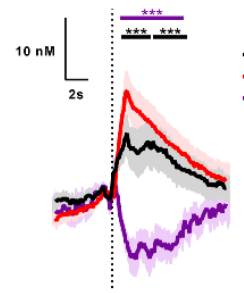

F

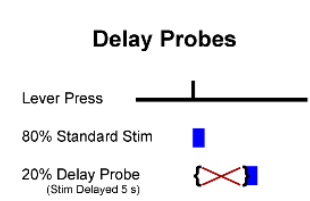

G

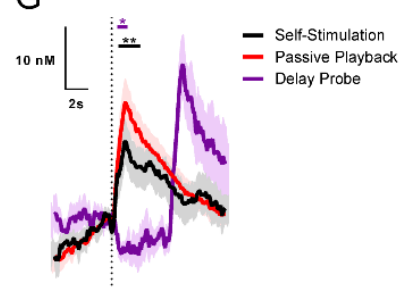

K

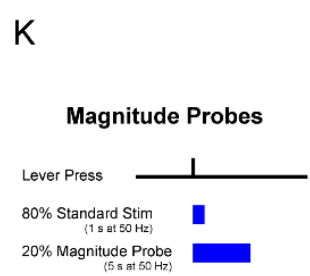

213

214

215

216

217

218

219

220

221

222

223

224

225

226

227

228

229

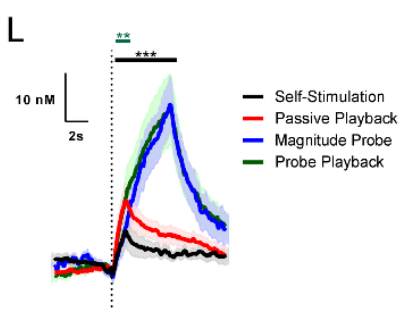

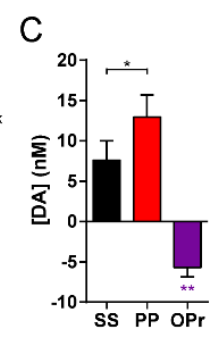
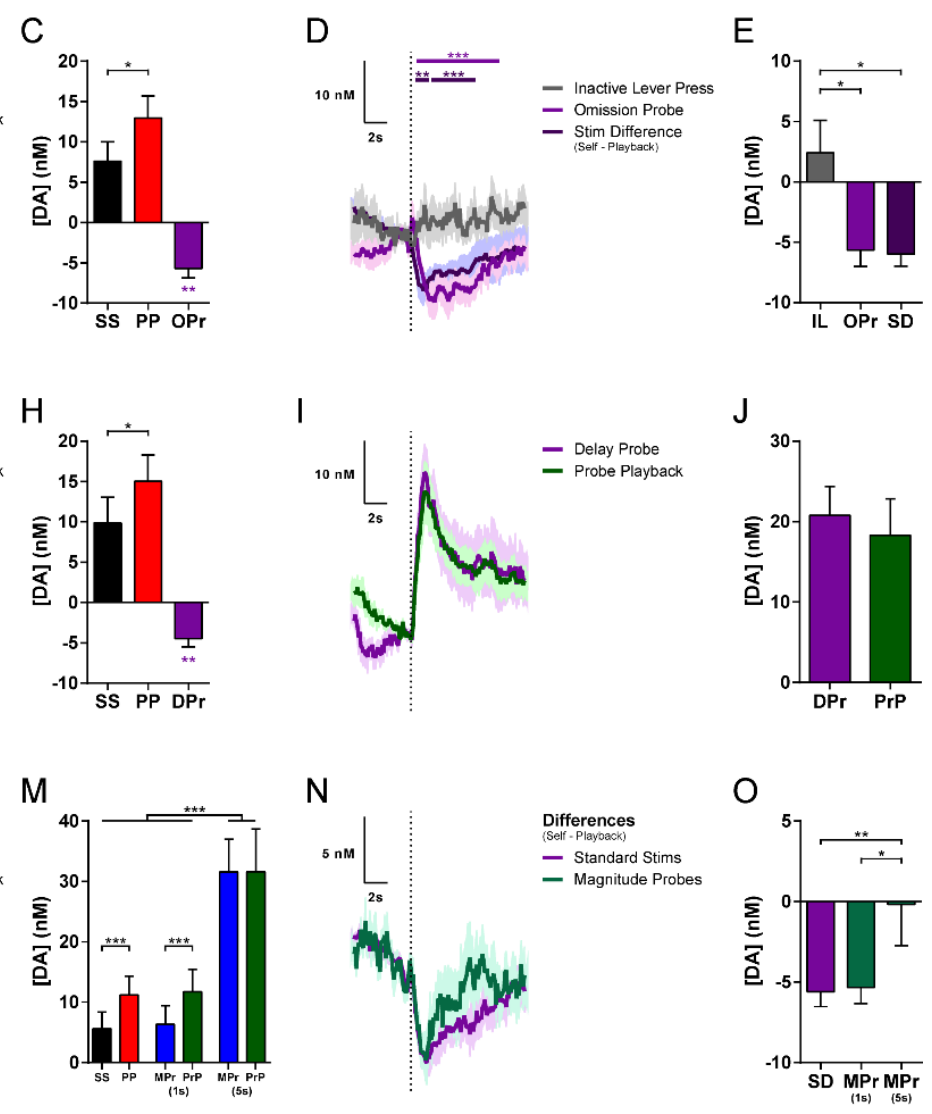

Figure 2. The Inhibition of Dopamine is Action-Specific and Temporally Precise. (A) Task schematic for Omission Probe sessions. (B) Mean change in dopamine concentration for stimulations and probes in Omission Probe sessions ( $n=8$ mice; permutation tests: Omission Probe vs. 0, magenta bar, $P=0.0001$; Self-Stimulation vs. Playback, black bar, $P=0.0003$ for first time cluster and $P=0.0001$ for second cluster). (C) Mean change in dopamine concentration in Omission Probe sessions (Self-Stimulation vs. Passive Playback, paired t test, $t_{7}=3.114, P=0.0170$; Omission Probe vs. 0 , one-sample $t$ test: $\left.t_{7}=4.810, P=0.0019\right)$. (D) Mean change in dopamine concentration for Inactive Lever presses, overlaid on Omission Probes from (B) and Difference traces (Self Stimulation minus Playback) from Fig. $1 \mathrm{H}(n=7$ mice; permutation tests: Inactive press vs. Omission Probe, magenta bar, $P=0.0001$; Inactive press vs. Stim Difference, purple bar, $P=0.007$ for first time cluster and $P=0.0007$ for second cluster. (E) Mean change in dopamine concentration for Inactive Lever presses versus Omission Probes from $(C)$ and stimulation Differences from Fig. 1 (one-way repeatedmeasures ANOVA, $F_{2,12}=7.419, P=0.0080$; Tukey's multiple comparisons tests: Inactive Lever vs. Omission Probe, $P=0.0133$; Inactive Lever vs. Stimulation Difference, $P=0.0174)$. (F) Task schematic for Delay Probe sessions. (G) Mean change in dopamine concentration for Standard 
Stimulations and probes in Delay Probe sessions ( $n=8$ mice; permutation tests: Delay Probe timeout period vs. 0 , magenta bar, $P=0.0103$; standard Self-Stimulation vs. Playback, black bar, $P=0.0001$ ). $(\mathbf{H})$ Mean change in dopamine concentration in Delay Probe sessions (SelfStimulation vs. Passive Playback, paired t test, $t_{7}=2.962, P=0.0210$; Delay Probe vs. 0 , onesample t test: $t_{7}=4.341, P=0.0034$ ). (I) Mean change in dopamine concentration for Delay Probes and Probe Playback, each aligned to stimulation onset. $(\mathbf{J})$ Mean change in dopamine concentration for Delay Probe stimulation and Probe Playback. (K) Task schematic for Magnitude Probe sessions. (L) Mean change in dopamine concentration for standard stimulations and probes in Magnitude Probe sessions ( $n=8$ mice; permutation tests: Magnitude Probe vs. Probe Playback, teal bar, $P=0.0052$; standard Self-Stimulation vs. Playback, black bar, $P=0.0001$ ). (M) Mean change in dopamine concentration in Magnitude Probe sessions (two-way repeated-measures ANOVA: main effect of Session Phase (Self vs. Playback), $F_{1,7}=$ $6.769, P=0.0353$; main effect of Stimulation Type (Standard, early and late Magnitude Probe), $F_{2,14}=31.32, P<0.0001$; Session Phase by Stimulation Type interaction, $F_{2,14}=7.724, P=$ 0.0055 ; Sidak's multiple comparisons tests: late Magnitude Probes vs. Standard Stimulations and vs. early Magnitude Probes within both Self and Playback phases, all $P \mathrm{~S}<0.0001$; Self vs. Playback for Standard Stimulations, $P=0.0006$; Self vs. Playback for early Magnitude Probes, $P=0.0008)$. (N) Difference traces: Self-Stimulation minus Passive Playback for Standard Stimulations and Magnitude Probes, from traces in (L). (O) Mean Differences comparing session phases (Self-Stimulation minus Passive Playback) for Standard Stimulations and Magnitude Probes at early $(1 \mathrm{~s})$ and late $(5 \mathrm{~s})$ time points from Difference traces in $(\mathrm{N})$. (Oneway repeated-measures ANOVA, $F_{2,14}=7.653, P=0.0057$; Tukey's multiple comparisons tests: Standard Stimulation Differences vs. late Magnitude Probe Differences, $P=0.0099$; early vs. late Magnitude Probe Differences, $P=0.0136$ ). SS, Self-Stimulation; PP, Passive Playback; OPr, Omission Probe; IL, Inactive Lever; SD, Standard Stimulation Difference (Self minus Playback); DPr, Delay Probe; PrP, Probe Playback; MPr, Magnitude Probe. See also Figure 2figure supplement 1 .

\section{Sequence-Specific Suppression of Nigrostriatal Dopamine Release}

In real life, goals are seldom achieved by a single action but instead mostly through a series of actions organized in spatiotemporal sequences (Gallistel, 1980; Jin and Costa 2010; Jin et al., 2014; Geddes et al., 2018). Having established that the observed prediction error-like suppression of nigrostriatal dopamine is action-specific and temporally restricted, we next turned to the question of whether such regulation of dopamine transmission reflects hierarchical control over learned action sequences (Lashley, 1951; Gallistel, 1980; Geddes et al., 2018). To this end, we trained a separate cohort of mice to perform a spatiotemporally heterogeneous action sequence, pressing the Left and then Right lever (LR) to earn optogenetic nigrostriatal dopamine neuron self-stimulation (See Methods; Figure 3A, Figure 3-figure supplement 1A-B). As mice increased the number of stimulations earned across days of training (Figure 3B), their behavior exhibited several indications of successfully learning this LR action sequence: They

271 lever press following each Left lever press and their probability of reinitiating with a Left lever 
272 press following each stimulation (Figure 3C). Their duration to complete these LR sequences

273 was shorter than the post-reinforcer reinitiation latency (Figure 3D), and the proportion of correct

274 LR sequences increased relative to other non-reinforced press pairs (Figure 3E). The total

275 presses per sequence and the number of consecutive presses on either lever both decreased

276 throughout training, collectively contributing to an increase in overall efficiency (Figure 3-figure

277 supplement $1 \mathrm{C}-\mathrm{F}$ ). Therefore, rather than simply associating the reinforcing outcome with the

278 most proximal action at the Right lever, the animals' behavior suggested that they indeed

279 concatenated the distinct action elements into chunked action sequences. Furthermore, the

280 mice significantly reduced their LR sequence performance during a contingency degradation

281 test (Figure 3-figure supplement 1G), indicating that these chunked action sequences also

282 were goal-directed.

284 We then recorded nigrostriatal dopamine transmission in these sequence-trained mice using the 285 same within-subject manipulation comparing Self-Stimulation versus Passive Playback-evoked

286 dopamine responses. We again found a robust suppression of the Self-Stimulated dopamine 287 response (Figure 3F-I, Figure 3-figure supplement $1 \mathrm{H}-\mathrm{I}$ ), recapitulating the main result from 288 the single-lever CRF cohort (Figure 1E-I). Importantly, no other combination of non-reinforced 289 press pairs caused inhibition comparable to the difference between the Self-Stimulated versus 290 Playback-evoked suppression of dopamine (Figure 3J-K), indicating that this inhibition was 291 specific to the learned action sequence. 

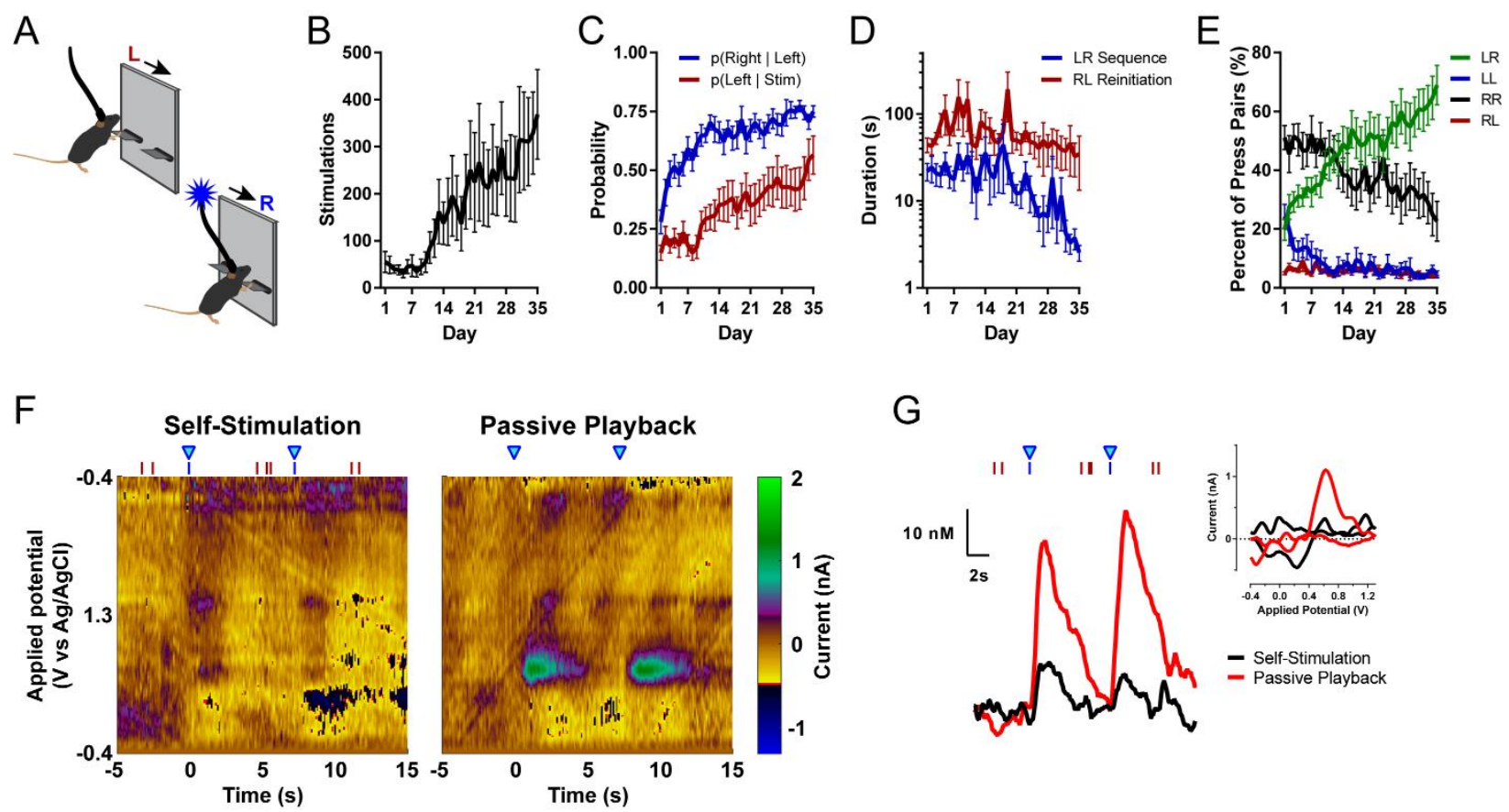

G

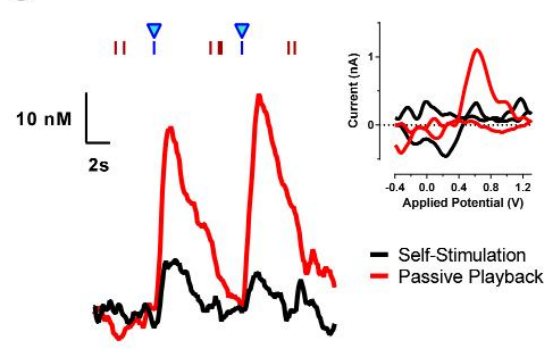

I
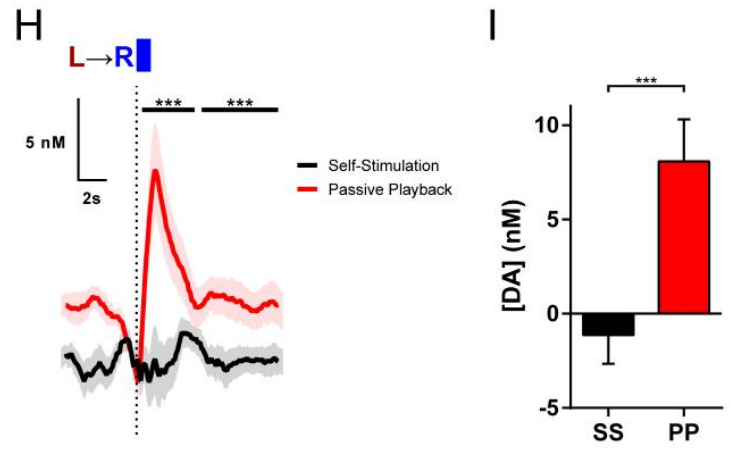

$\mathrm{J}$

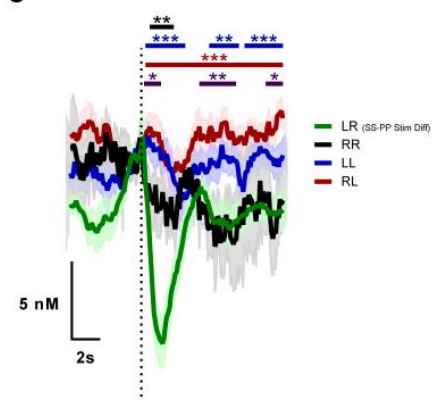

$\mathrm{K}$

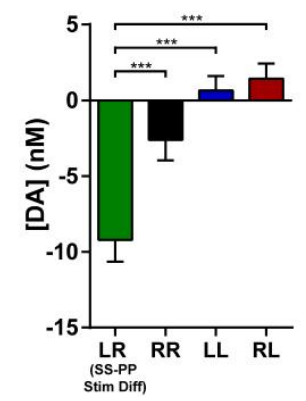

Figure 3. Sequence-Specific Inhibition of Nigrostriatal Dopamine during Performance of Learned LR Sequence. (A) Left-Right Sequence Self-Stimulation task schematic. (B) Stimulations earned across days of training ( $n=13$ mice; one-way repeated-measures ANOVA: $\left.F_{34,408}=3.550, P<0.0001\right)$. (C) Transition probabilities: probability of pressing Right lever after each Left lever press, and probability of reinitiating Left press after stimulation (two-way repeated-measures ANOVA: main effect of Day, $F_{34,408}=8.838, P<0.0001$; main effect of Transition Type, $F_{1,12}=47.18, P<0.0001$; Dunnett's multiple comparisons tests vs. Day 1: $p$ (Right | Left) first significantly differs on Day 3, $P=0.0266$; $p$ (Left | Stim) first significantly differs on Day 14, $P=0.0265$ ). (D) Median latencies to complete Left-Right sequences and to reinitiate a new sequence after previous stimulation (two-way repeated-measures ANOVA: main effect of Interval Type, $F_{1,12}=18.79, P=0.0010$ ). (E) Relative frequency of each combination of lever press pairs (two-way repeated-measures ANOVA: main effect of Pair Type, $F_{3,36}=32.58$, $P<0.0001$; Pair Type by Day interaction, $F_{102,1224}=4.315, P<0.0001$ ). (F) Representative voltammetric pseudocolor plots during two Left-Right sequences for optogenetic intracranial Self-Stimulation (left) and corresponding stimulations from the Passive Playback phase (right). 
Mean dopamine concentration changes evoked by stimulation in each phase $(n=12$ mice; permutation test, $P S=0.0001$ for both time clusters). (I) Mean change in dopamine concentration (paired t test, $t_{11}=6.403, P<0.0001$ ). (J) Mean dopamine concentration changes evoked by non-stimulated pairs of lever presses (5-s maximum inter-press interval within pair). Traces are aligned to the second press in each pair type. The LR sequence stimulation Difference (Self-Stimulation minus Passive Playback) is overlaid for comparison (green). (Permutation tests: LR Stim Difference vs. RR, black bar, $P=0.0046$; LR Stim Difference vs. LL, blue bars, $P s=0.0002$ for first time cluster, 0.0017 for second, and 0.0007 for third cluster; LR Stim Difference vs. $\mathrm{RL}$, maroon bar, $P=0.0001$; RR vs. $\mathrm{RL}$, purple bars, $P \mathrm{~s}=0.0185$ for first time cluster, 0.0021 for second, and 0.0163 for third cluster). (K) Mean change in dopamine concentration for each combination of non-reinforced press pairs during the Self-Stimulation phase and the LR sequence stimulation Difference (Self minus Playback). (One-way repeatedmeasures ANOVA, $F_{3,33}=20.34, P<0.0001$; Tukey's multiple comparisons tests: LR Stim Difference vs. RR, $P=0.0007$; LR Stim Difference vs. LL and vs. RL, $P S<0.0001)$. Stim, Stimulation; LR, Left-Right sequence; RR, Right-Right; LL, Left-Left; RL, Right-Left; SS, SelfStimulation; PP, Passive Playback; Diff, Difference. See also Figure 3-figure supplement 1.

\section{Differential Regulation of Dopamine by Individual Actions within Learned Sequence}

Beyond this sequence-type specificity, we further examined the question of whether dopamine transmission might reflect regulation at the level of individual action elements or instead at a higher sequence level in a hierarchy of behavioral control. For example, if regulated with each action element, we might expect similar inhibition for each individual Left and Right lever press, and summation of each to the full inhibition at outcome delivery. Alternatively, since animals chunked these action elements into fully concatenated action sequences, we might expect the action-induced inhibition of dopamine to begin at sequence initiation and persist throughout performance of this chunked action sequence. The results were inconsistent with either of these hypotheses, instead exhibiting a distinct form of sequence-specificity consistent with hierarchical control (Jin and Costa 2015; Geddes et al., 2018). Initiating Left lever presses did not cause any inhibition of dopamine, instead revealing a slight, albeit non-significant increase in dopamine release (Figure 4A-B). Similarly, additional recording sessions with probe stimulations delivered on $20 \%$ of initiating Left presses revealed no inhibition of dopamine evoked by these Left Probes (Figure 4C-H). Instead, these Left Probes actually evoked significantly greater dopamine release than their Playback (Figure 4F). At the individual animal level, 5 out of 8 mice (62.5\%)

343 showed a significant dopamine increase, and none showed a significant suppression. Outside of

344 LR sequences, single Right lever presses (see Methods) did not result in inhibition of dopamine, 345 in stark contrast with the full inhibition of Self-Stimulated versus Playback-evoked dopamine for 346 correct LR sequences (Figure 4I-J). The dopamine response to Probe stimulations for these 347 isolated Right presses also did not differ from their Playback, exhibiting no significant inhibition 
348 (Figure 4K-P). This same action at the Right lever therefore reveals highly distinct regulation of 349 dopamine dynamics depending on the action's membership within the learned sequence or not.

350 These results indicate that the dopaminergic prediction errors are selective to the learned action 351 sequence and reflect sequence-level hierarchical control over instrumental behavior.

A

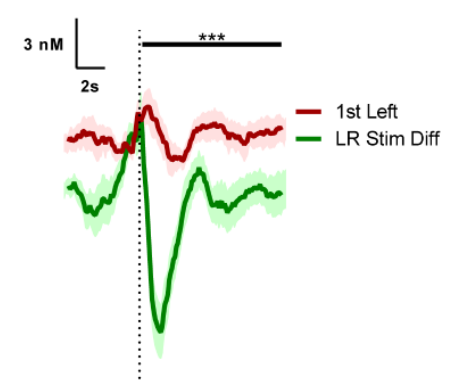

$\mathrm{E}$

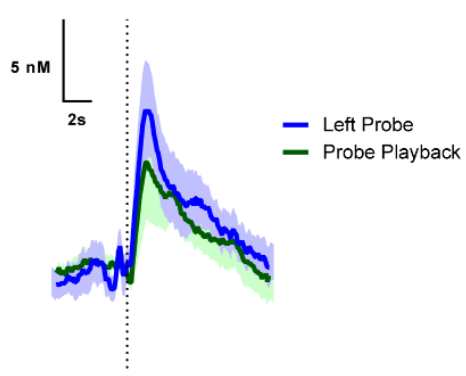

I

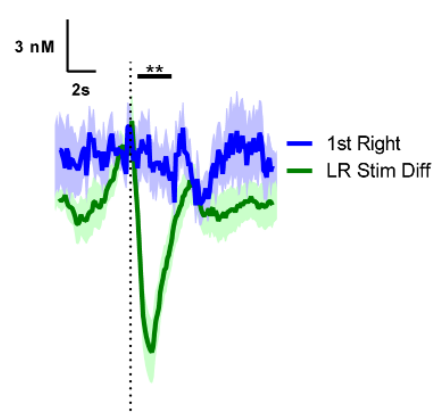

M

352

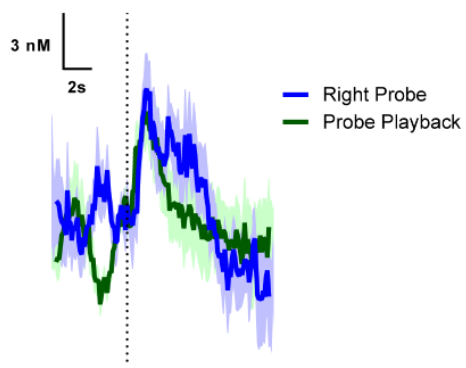

B

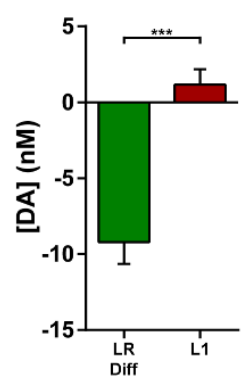

$\mathrm{F}$

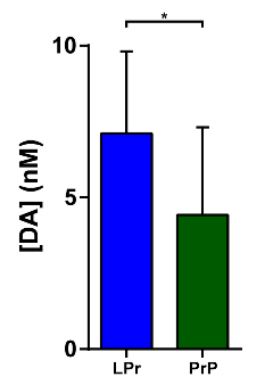

J

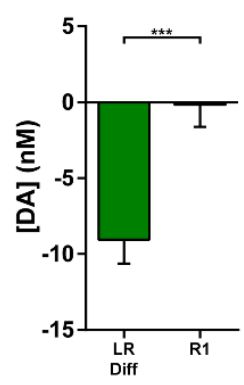

$\mathrm{N}$

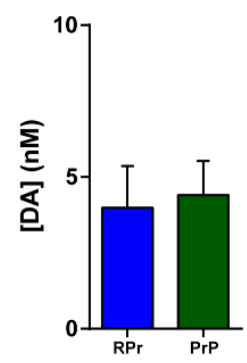

C ${ }_{(20 \%)} \mathrm{L}$

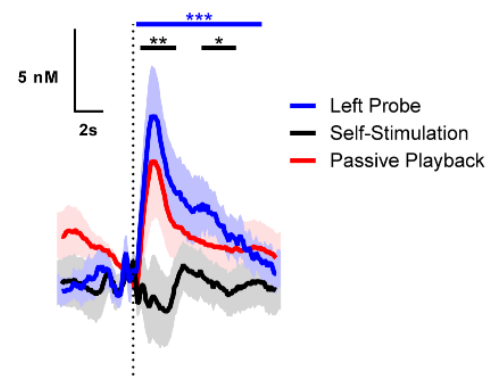

G

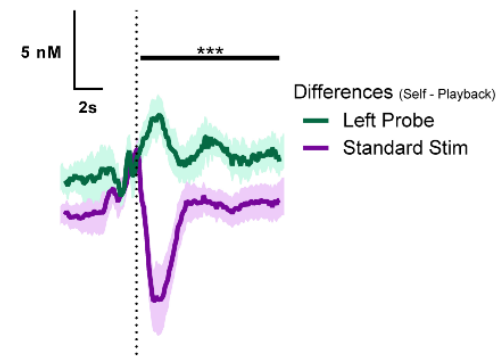

${ }^{(50 \%)} \mathrm{R} \square$
$\mathrm{L} \rightarrow \mathrm{R} \square$

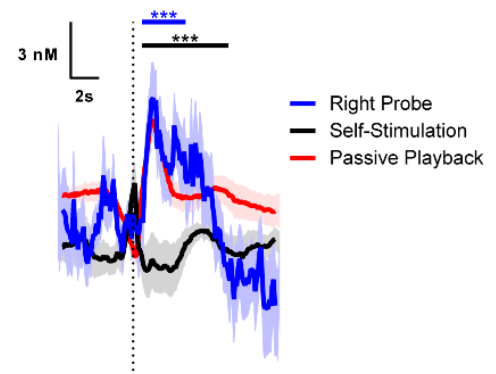

O

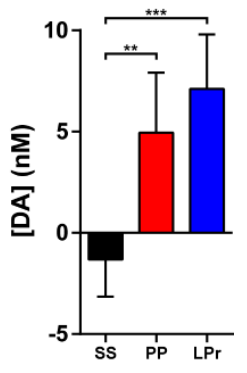

$\mathrm{H}$

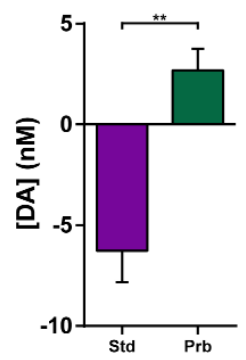

L

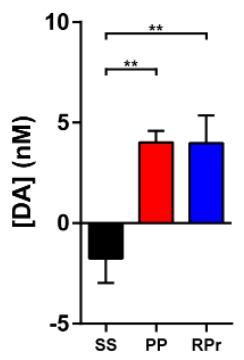

P

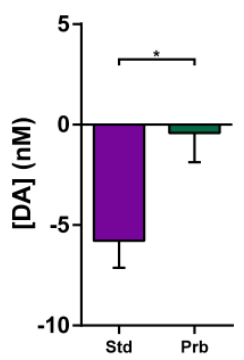


Figure 4. Different Regulation of Dopamine by Individual Actions of the LR Sequence. (A) Mean dopamine concentration change to first Left lever press following previous stimulation during Left-Right sequence task performance, overlaid with the Difference Trace (Self minus Playback) for LR sequence stimulations from Fig. 3J for comparison ( $n=12$ mice; permutation test, $P=0.0001$ ). (B) Mean change in dopamine concentration for first Left lever presses and LR sequence stimulation Difference $\left(t_{11}=6.325, P<0.0001\right)$. (C) Mean dopamine concentration changes in Left-Right sequence sessions with Left Lever Probes $(n=8$ mice; permutation tests: Left Probe vs. Standard LR Self-Stimulation, blue bar, $P=0.0001$; Standard LR Self-Stimulation vs. Playback, black bars, $P S=0.0049$ for first and 0.011 for second time clusters, respectively). (D) Mean change in dopamine concentration in Left Probe sessions (one-way repeatedmeasures ANOVA, $F_{2,14}=17.19, P=0.0002$; Tukey's multiple comparisons tests: Left Probe vs. LR Self-Stimulation, $P=0.0002$; LR Self-Stimulation vs. Playback, $P=0.0024)$. (E) Mean dopamine concentration changes in Left Probes and the Probe Playback. (F) Mean change in dopamine concentration to Left Probes and Probe Playback (paired t test, $t_{7}=2.519, P=$ 0.0399). (G) Difference traces from Left Probe Sessions: Self-Stimulation minus Passive Playback for Standard LR Stimulations and Left Probes, from traces in $(C)$ and $(E)$; (permutation test, $P=0.0001$ ). (H) Mean Differences comparing session phases (Self-Stimulation minus Passive Playback) for Standard LR Stimulations and Left Probes (paired t test, $t_{7}=5.125, P=$ 0.0014). (I) Mean dopamine concentration change to first Right lever press following previous stimulations, overlaid with the Difference Trace (Self minus Playback) for LR sequence stimulations from Fig. $3 \mathrm{~J}$ for comparison. First Right press was an additional press on the Right lever after a previous stimulation, without approaching the Left lever $(n=11$ mice; permutation test, $P=0.0012)$. (J) Mean change in dopamine concentration for first Right lever presses and LR sequence stimulation Difference $\left(t_{10}=5.690, P=0.0002\right)$. (K) Mean dopamine concentration changes in Left-Right sequence sessions with Right Lever Probes where animal did not approach the Left lever in the preceding inter-stimulation interval $(n=10$ mice; permutation tests: Right Probe vs. Standard LR Self-Stimulation, blue bar, $P=0.0001$; Standard LR SelfStimulation vs. Playback, black bar, $P=0.0001)$. (L) Mean change in dopamine concentration in Right Probe sessions (one-way repeated-measures ANOVA, $F_{2,18}=10.47, P=0.0010$; Tukey's multiple comparisons tests: Right Probe vs. LR Self-Stimulation, $P=0.0026$; LR Self-

Stimulation vs. Playback, $P=0.0024)$. (M) Mean dopamine concentration changes in Right Probes and the Probe Playback. (N) Mean change in dopamine concentration to Right Probes and Probe Playback. (0) Difference traces from Right Probe Sessions: Self-Stimulation minus Passive Playback for Standard LR Stimulations and Right Probes, from traces in $(K)$ and $(M)$; (permutation test, $P=0.0002)$. (P) Mean Differences comparing session phases (SelfStimulation minus Passive Playback) for Standard LR Stimulations and Right Probes (paired t test, $t_{9}=3.080, P=0.0131$ ). LR Stim Diff, Left-Right Stimulation Difference (Self minus Playback); L1, $1^{\text {st }}$ Left Press; R1, $1^{\text {st }}$ Right Press; SS, Self-Stimulation; PP, Passive Playback; LPr, Left Probe; PrP, Probe Playback; Std, Standard LR Stimulation; Prb, Probe; RPr, Right Probe.

\section{DISCUSSION}

Overall, we have demonstrated that nigrostriatal dopamine transmission to reinforcing outcomes is strongly suppressed when this outcome is the expected consequence of the animal's own action. This inhibition of outcome-evoked dopamine following self-initiated actions parallels 
commonly observed reward prediction errors in explicit stimulus-outcome and stimulusresponse behavioral contexts. The current results therefore expand this phenomenon to include action-outcome prediction errors that support instrumental associations underlying self-initiated goal-directed behavior. This action-outcome prediction error was specific to the typically reinforced action, temporally restricted to counteract the expected consequence of that action, and exhibited sequence selectivity consistent with a high level of hierarchical control over

405 chunked action sequences. The prediction errors signaled by dopamine transmission therefore

406 reflect not only expectations associated with Pavlovian cues or behavioral responses to such

407 discrete stimuli, but also the expected outcomes of self-initiated instrumental actions and

408 sequences. Compelling behavioral and neural evidence for action chunking also is well

409 established (Graybiel, 1998; Hikosaka, 1998; Matsumoto et al., 1999; Barnes et al., 2005; Jin

410 and Costa, 2010; Wassum et al., 2012; Jin et al., 2014; Jin and Costa 2015; Collins et al., 2016;

411 Geddes et al., 2018), but the mechanisms subserving such sequence learning remain poorly

412 understood. While the exact role of nigrostriatal dopamine throughout sequence acquisition

413 requires further direct investigation, the current results demonstrate that the performance of

414 well-learned action sequences entails distinct dopamine dynamics for actions within these

415 sequences. That nigrostriatal dopamine transmits specific action-outcome prediction errors and

416 exhibits sequence-dependent hierarchical regulation provides critical new insight into these

417 important neuromodulatory dynamics in goal-directed behavioral control, an under-examined

418 domain of instrumental action beyond spontaneous movement of unknown purpose and

419 responding to reward-predictive cues.

421 Several aspects of our opto-ICSS experimental design conferred distinct advantages for 422 examining the regulation of nigrostriatal dopamine dynamics in goal-directed behavior. The 423 current study used an entirely within-subject design and direct optogenetic excitation to 424 selectively stimulate dopamine neurons and record dopamine transmission at identical locations 425 within a given animal, in contrast to previous ICSS studies that used non-selective electrical 426 stimulation of the midbrain and compared dopamine release between trained versus naïve 427 animals (Garris et al., 1999; Kilpatrick et al., 2000) or did not include temporally matched non428 contingent playback (Owesson-White et al., 2008; Rodeberg et al., 2016; Covey and Cheer, 429 2019). Traditional procedures with natural reward invariably require additional consummatory 430 actions such as magazine approach or licking for reward retrieval, which itself might regulate 431 dopamine dynamics and complicate the data analyses. Although selective optogenetic 432 stimulation lacks the specific sensory features such as flavor that typically define the identity of 
433 natural reward outcomes (Kruse et al., 1983; Corbit and Janak, 2007; Collins et al., 2016;

434 Takahashi et al., 2017; Keiflin et al., 2018; Balleine, 2019), the direct intracranial delivery

435 permitted precise temporal control over outcome receipt. Furthermore, direct optogenetic

436 stimulation also bypasses afferent circuitry representing any natural reward itself, permitting the

437 current focus on regulation of dopamine by specific action-associated expectancies. These

438 features of the current design collectively yielded results consistent with nigrostriatal dopamine

439 transmitting an action-outcome prediction error signal.

Although direct optogenetic stimulation indeed approaches an essentially identity-less outcome

442 (Wise, 2002), this outcome delivery does coincide with sensory feedback during the action, such

443 as somatosensory contact or auditory feedback from pressing the lever. However, these

444 sensory reafferents are comparable for inactive lever presses or other non-reinforced action

445 sequences, and therefore cannot account for the selective suppression of dopamine evoked as

446 the consequence of reinforced actions (Figures 2D-E and 3J-K). Indeed, the distinct regulation

447 of dopamine to the same action depending on sequence membership (Figure 4I-P) again

448 provides clear evidence that the observed suppression was due to specific action expectancies

449 rather than sensory feedback. Whereas the suppression of outcome-evoked dopamine release

450 is therefore unlikely accounted for by different sensory features between the session phases,

451 this action-induced suppression may instead share important commonalities with efference copy

452 (or corollary discharge) phenomena widely observed in numerous other sensorimotor systems

453 throughout the nervous systems of many different species (Wolpert et al., 1995; Crapse and

454 Sommer, 2008; Schneider et al., 2018; Wurtz, 2018). Indeed, the current results provide

455 evidence that a learned, sequence-level efference copy can suppress the neurochemical

456 consequence of the complete action sequence, distinct from the regulation by individual action

457 elements. These findings align with the recent demonstration of dopaminergic prediction errors

458 for evaluating sequential sensorimotor control relative to internal performance templates

459 (Gadagkar et al., 2016), and are broadly consistent with the prominent role proposed for

460 efference copies in striatal-dependent learning (Redgrave and Gurney, 2006; Fee, 2014).

462 The current study's recordings targeted the dorsomedial striatum (DMS), which is widely

463 implicated in goal-directed instrumental behavior (Yin et al., 2005; Yin et al., 2008; Gremel \&

464 Costa, 2013; Balleine, 2019; Matamales et al., 2020). A natural next question is whether

465 regulation of dopamine dynamics differs in other striatal subregions. Recent work found an

466 attenuation of mesolimbic dopamine release in the nucleus accumbens core during self-paced 
opto-ICSS of ventral tegmental area dopamine neurons, albeit without comparison to temporally matched non-contingent playback stimulation (Covey \& Cheer, 2019). Together, this finding and the present study extend earlier work reporting suppression of both mesolimbic (Garris et al., 1999) and nigrostriatal dopamine (Kilpatrick et al., 2000) evoked by non-selective electrical selfstimulation in trained animals versus non-contingent playback in naïve animals. Further, in a

472 discrete-trial, cued task variant, Covey and Cheer (2019) also found an attenuation of

473 optogenetically stimulated dopamine release and a concomitant increase in cue-evoked

474 release, consistent with classic reward prediction errors in natural reward contexts (Schultz et

475 al., 1997). Indeed, another recent study found predominant prediction-error responses in

476 dopamine axonal activity throughout much of the ventral, dorsomedial, and dorsolateral striatum

477 (DLS) in a cued discrimination task for water reward (Tsutsui-Kimura et al., 2020). Here, a

478 notable difference in the DLS was a lack of dips below baseline despite similarly suppressive

479 effects of reward expectation across regions. Based on these collective findings, we therefore

480 would predict that most effects observed within the DMS in the current study would be largely

481 similar in the accumbens core (Covey \& Cheer, 2019), and although we also would expect

482 suppression in the DLS, we also might not expect negative prediction errors to cause dips below

483 baseline there (Tsutsui-Kimura et al., 2020). In contrast, the predictions are perhaps less clear

484 for aspects of the accumbens shell and the caudal-most tail of the striatum, where distinct and

485 surprising dopamine dynamics have been revealed particularly in aversive domains (de Jong et

486 al., 2018; Menegas et al., 2018; Steinberg et al., 2020). Overall, potential heterogeneity of

487 dopamine signaling across striatal subregions remains an important topic of investigation.

489 Uncovering the circuit mechanisms responsible for this dopaminergic action-outcome prediction 490 error also remains an important open question for future research. The current results constrain 491 candidate mechanisms to those with fairly rapid onset, transient duration, and sufficiently strong 492 inhibition to suppress or shunt even direct optogenetic depolarization. Nigrostriatal dopamine 493 neurons receive monosynaptic inputs from all basal ganglia nuclei (Watabe-Uchida et al., 2012;

494 Lerner et al., 2015; Menegas et al., 2015), the majority of which are predominantly inhibitory

495 GABAergic projections (Tepper and Lee, 2007; Brazhnik et al., 2008; Evans et al., 2020).

496 Striatal, pallidal, and nigral basal ganglia nuclei contain many cells exhibiting prominent activity

497 related to action sequence initiation, termination, and transitions (Jin and Costa, 2010; Jin et al.,

498 2014; Geddes et al., 2018), as well as action-outcome value information (Samejima et al., 2005;

499 Lau and Glimcher 2008; Hong and Hikosaka, 2008; Roesch et al., 2009; Tachibana and

500 Hikosaka, 2012; Kim et al., 2017) that may converge and contribute to these dopamine neuron 
501 computations. Recent studies have suggested that dynamic nigrostriatal dopamine might

502 regulate ongoing actions (Jin and Costa, 2010; Barter et al. 2015; Panigrahi et al. 2015; da Silva

503 et al. 2018) and bias online action selection (Howard et al. 2017). The current results revealed

504 that nigrostriatal dopamine can encode action-outcome prediction errors critical for action

505 learning. Together they underscore the importance of dopamine for action selection at short as

506 well as long timescales, and have important implications in many neurological disorders such as

507 Parkinson's disease, schizophrenia, and addiction. 
MATERIALS AND METHODS

\begin{tabular}{|c|c|c|c|c|}
\hline \multicolumn{5}{|c|}{ Key Resources Table } \\
\hline $\begin{array}{l}\text { Reagent } \\
\text { type } \\
\text { (species) } \\
\text { or resource }\end{array}$ & Designation & $\begin{array}{l}\text { Source or } \\
\text { reference }\end{array}$ & Identifiers & $\begin{array}{l}\text { Additional } \\
\text { information }\end{array}$ \\
\hline $\begin{array}{l}\text { genetic } \\
\text { reagent } \\
\text { (Mus } \\
\text { musculus) }\end{array}$ & DAT-cre (S/c6a3) & $\begin{array}{l}\text { Jackson } \\
\text { Laboratory }\end{array}$ & $\begin{array}{l}\text { RRID:IMSR_JAX:02 } \\
0080\end{array}$ & \\
\hline $\begin{array}{l}\text { genetic } \\
\text { reagent } \\
\text { (Mus } \\
\text { musculus) }\end{array}$ & $\begin{array}{l}\text { Ai32 (RCL- } \\
\text { ChR2(H134R)/eY } \\
\text { FP) }\end{array}$ & $\begin{array}{l}\text { Jackson } \\
\text { Laboratory }\end{array}$ & $\begin{array}{l}\text { RRID:IMSR_JAX:02 } \\
4109\end{array}$ & \\
\hline $\begin{array}{l}\text { strain, strain } \\
\text { background } \\
\text { (Adeno- } \\
\text { associated } \\
\text { virus) }\end{array}$ & $\begin{array}{l}\text { AAV5-EF1a-DIO- } \\
\text { ChR2(H134R)- } \\
\text { mCherry }\end{array}$ & UNC vector core & RRID:SCR_002448 & \\
\hline antibody & $\begin{array}{l}\text { anti-tyrosine } \\
\text { hydroxylase } \\
\text { (rabbit polyclonal) }\end{array}$ & Abcam & $\begin{array}{l}\text { Cat\#ab112; } \\
\text { RRID:AB_297840 }\end{array}$ & $(1: 1000)$ \\
\hline antibody & $\begin{array}{l}\text { anti-GFP (chicken } \\
\text { polyclonal) }\end{array}$ & Novus Biologicals & $\begin{array}{l}\text { Cat\#NB100-1614; } \\
\text { RRID:AB_10001164 }\end{array}$ & $(1: 1000)$ \\
\hline antibody & $\begin{array}{l}\text { anti-rabbit Cy3 } \\
\text { (donkey } \\
\text { polyclonal) }\end{array}$ & $\begin{array}{l}\text { Jackson } \\
\text { ImmunoResearch }\end{array}$ & $\begin{array}{l}\text { Cat\#711-165-152; } \\
\text { RRID:AB_2307443 }\end{array}$ & $(1: 250)$ \\
\hline antibody & $\begin{array}{l}\text { anti-chicken Alexa } \\
\text { Fluor } 488 \text { (donkey } \\
\text { polyclonal) }\end{array}$ & $\begin{array}{l}\text { Jackson } \\
\text { ImmunoResearch }\end{array}$ & $\begin{array}{l}\text { Cat\#703-545-155; } \\
\text { RRID:AB_2340375 }\end{array}$ & $(1: 250)$ \\
\hline
\end{tabular}

\section{Animals}

513 All procedures were approved by the Institutional Animal Care and Use Committee at the Salk

514 Institute for Biological Studies and were conducted in accordance with the National Institute of

515 Health's Guide for the Care and Use of Laboratory Animals. Experiments were performed using

516 male and female mice, at least two months old, group-housed (2-5 mice / cage) on a $12 \mathrm{hr}$

517 light/dark cycle (lights on at 6:00 am). DAT-cre mice (Jackson Laboratory \# 020080; Zhuang et 
al., 2005) were either crossed with the Ai32 line (RCL-ChR2(H134R)-EYFP, Jackson Laboratory \# 024109; Madisen et al., 2012) or injected with cre-dependent AAV in the SNc to selectively express channelrhodopsin-2 in their dopamine neurons.

\section{Surgical Procedures}

Mice were anesthetized with isoflurane (3\% induction, $0.5-1.5 \%$ sustained), their head shaved, and they were placed in a stereotaxic frame. The scalp was swabbed with $70 \%$ isopropyl alcohol and a povidine-iodine solution, and given a subcutaneous injection of bupivicaine (2 $\mathrm{mg} / \mathrm{kg}$ ) for local anesthesia. After a midline incision and leveling the skull, skulls were dried and coated with OptiBond adhesive and/or implanted with skull screws. Craniotomies were drilled over the dorsal striatum (+ 0.5-0.8 mm AP, $1.5 \mathrm{~mm} \mathrm{ML}$ from bregma) for the voltammetric working electrode, the substantia nigra pars compacta (SNc: -3.1-3.3 mm AP, 1.3 mm ML) for the fiber optic(s), and an arbitrary distal site for the $\mathrm{Ag} / \mathrm{AgCl}$ reference electrode. For DAT-cre mice not already crossed with the Ai32 line, $300 \mathrm{nl}$ of AAV5-EF1a-DIO-ChR2(H134R)-mCherry (UNC vector core) was injected into the SNc (4.1 mm ventral from dura; $100 \mathrm{nl} / \mathrm{min}$ ), and the injection needle was left in place for 5 min before being slowing withdrawn (Howard et al., 2017). For all FSCV mice, the $\mathrm{Ag} / \mathrm{AgCl}$ reference was inserted under the skull and cemented in place, and a carbon-fiber microelectrode (Clark, Sandberg et al., 2010) was lowered into the striatum (2.3-2.5 mm DV from dura) while applying a voltammetric waveform (see FSCV section) at $60 \mathrm{~Hz}$ for $10-15 \mathrm{~min}$, and then at $10 \mathrm{~Hz}$ until the background had stabilized. A fiber optic (200 $\mu \mathrm{m}$ core; Sparta et al., 2011; Howard et al. 2017) was lowered targeting the ipsilateral SNc (3.8-4.1 mm DV). DAT-cre $x$ Ai32 mice received 1-s 50- $\mathrm{Hz}$ optical stimulation while striatal dopamine was recorded with FSCV to ensure electrode functionality and fiber placement. Mice

543 subsequently trained in the Left-Right sequence task cohort (see Behavioral Training) also were

544 implanted with a fiber optic over the contralateral SNc for bilateral stimulation. All implants were

545 cemented to the skull along with a connector from the reference and working electrodes for later

546 attachment to the FSCV head-mounted amplifier (headstage). For electrophysiological

547 identification of dopamine neurons, DATcre $x$ Ai32 mice were implanted unilaterally in the SNc

548 with an electrode array (Innovative Neurophysiology) with 16 tungsten contacts (2 x 8), $35 \mu \mathrm{m}$ in

549 diameter, spaced $150 \mu \mathrm{m}$ apart within rows and $200 \mu \mathrm{m}$ apart between rows. The array had a

550 fiber optic directly attached, positioned $\sim 300 \mu \mathrm{m}$ from the electrode tips, to permit coupling to

551 the laser for stimulation delivery (Jin and Costa 2010; Howard et al. 2017). The silver grounding 
552 wire was attached to a skull screw, and the array was affixed with dental cement. Mice received

553 buprenorphine (1 mg/kg, s.c.) for analgesia and dexamethasone (2.5 mg/kg, s.c.) or ibuprofen in

554 their drinking water for post-operative anti-inflammatory treatment, recovered in a clean home

555 cage on a heating pad, were monitored daily for at least 3 days, and allowed to recover for at

556 least 10 days before beginning behavioral training.

\section{Behavioral Training}

Behavioral training was conducted in standard operant chambers (Med Associates) inside sound attenuating box, as previously described (Howard et al., 2017; Geddes et al., 2018). Mice were connected to the fiber optic patch cable from the laser (LaserGlow; $473 \mathrm{~nm}, \sim 5 \mathrm{~mW}$ measured before each session) and placed in the operant chamber, and optogenetic intracranial self-stimulation (opto-ICSS) sessions began with the insertion of two levers and the onset of a central house light on the opposite wall. The levers remained extended and the house light remained on for the duration of the $60 \mathrm{~min}$ sessions.

\section{Continuous reinforcement cohort}

570 Each press on the designated Active lever resulted in $1 \mathrm{~s}$ of optical stimulation $(50 \mathrm{~Hz}, 10 \mathrm{~ms}$ pulse width) on a continuous reinforcement (CRF) schedule, other than additional presses

572 during an ongoing stimulation train, which were recorded but had no consequence. Presses on 573 the other, Inactive lever also were recorded but had no consequence. The sides of the Active 574 and Inactive levers were counterbalanced relative to both the operant chamber and implanted 575 hemisphere across mice, and remained fixed across training days for a given animal. Once 576 mice reliably made at least 100 Active lever presses per session for 3 consecutive days, they also were connected to a voltammetry headstage before each session to allow habituation to behaving with this additional tethering. If a mouse failed to interact with the levers during its first 3 days of training, it was placed on food restriction overnight and a sucrose pellet was placed on the lever during its next behavioral session to encourage exploration. Once mice were reliably pressing the Active lever, they remained on ad libitum access to food and water in their home

582 cages for all subsequent behavioral training and FSCV recordings. Mice were trained for at least

5833 days while tethered to the FSCV headstage and meeting the behavioral criteria of at least 100

584 Active lever presses before FSCV recordings commenced (mean \pm SEM $=11.1 \pm 1.2$ training 585 days). 


\section{Left-Right sequence cohort}

588 Mice in the Left-Right (LR) sequence cohort were initially trained on single-lever CRF opto-

589 ICSS. For this cohort's CRF training, only one lever was extended in each of two 30-min blocks

590 per session (order counterbalanced across mice), and presses in both left- and right-lever

591 blocks yielded the same 1-s, 50- $\mathrm{Hz}$ stimulation. To expedite this initial training stage, all mice in

592 this cohort were food restricted prior to their first session, and were maintained at $85 \%$ their

593 free-feeding baseline weight with $\sim 2.5 \mathrm{~g}$ of standard lab chow per mouse in their home cage

594 after the daily training sessions. Once mice made at least 100 presses in each block for 3

595 consecutive days, they were returned to ad libitum food access in their home cage, CRF training

596 continued until they again met this 100-press criterion for another 3 days, and they then began

597 training on the LR sequence task.

In LR sequence session, both levers were inserted at the start of the session and remained

600 extended for the duration of the 60 min sessions. To receive stimulation $(1 \mathrm{~s}, 50 \mathrm{~Hz})$, mice now

601 had to press the Left and then Right lever. No other combination of lever press pairs (Left-Left,

602 Right-Right, or Right-Left) was reinforced with stimulation. After reaching the behavioral criterion

603 of receiving at least 100 stimulations per session for 3 consecutive days, mice were habituated

604 to tethering with the FSCV headstage, and received further training while tethered until they

605 again met this 100-stimulation 3-day criterion and FSCV recording sessions commenced (mean

$606 \pm \mathrm{SEM}=56.9 \pm 7.6$ training days). A subset of animals was trained under the same procedures

607 to instead perform the Right-Left sequence as a spatial control, but we refer to the LR sequence

608 throughout for simplicity. The hemisphere of the implanted FSCV recording electrode also was

609 counterbalanced relative to this sequence direction across mice.

\section{Contingency degradation}

612 A contingency degradation test session began with $30 \mathrm{~min}$ of standard opto-ICSS (CRF for the

613 CRF cohort, LR sequence task for the LR cohort). In the subsequent 30-min contingency

614 degradation test phase, the levers remained extended, but stimulation was decoupled from task

615 performance and instead was delivered regardless of whether the mice pressed any levers

616 (Witten, Steinberg, et al., 2011; Koralek, Jin, et al., 2012; Clancy et al., 2014; Neely et al.,

617 2018). For each mouse, the timing of these non-contingent stimulations during the test phase

618 was matched to the time stamps of stimulations earned during that animal's preceding opto-

619 ICSS phase in the first half of the session, ensuring that the stimulation rate and distribution of 
620 inter-stimulation intervals were yoked within-subject to a given animal's own opto-ICSS

621 performance.

\section{Fast-Scan Cyclic Voltammetry (FSCV)}

626 Striatal dopamine was recorded with in vivo FSCV in behaving animals as previously described

627 (Clark et al., 2010; Hollon et al. 2014; Howard et al., 2017). Briefly, voltammetric waveform

628 application consisted of holding the potential at the carbon-fiber electrode at $-0.4 \mathrm{~V}$ relative to

629 the $\mathrm{Ag} / \mathrm{AgCl}$ reference between scans, and ramping to $+1.3 \mathrm{~V}$ and then back to $-0.4 \mathrm{~V}$ at $400 \mathrm{~V} / \mathrm{s}$

630 for each scan. Prior to the initial FSCV recording during opto-ICSS performance, this

631 voltammetric waveform was applied at $60 \mathrm{~Hz}$ for at least one hour while mice were in a 'cycling

632 chamber' outside the operant box, then at $10 \mathrm{~Hz}$ until the background current had stabilized.

633 Mice then received experimenter-delivered optical stimulations (1 s, $50 \mathrm{~Hz}$ ) to ensure electrode

634 functionality.

636 For opto-ICSS sessions with FSCV recordings, electrodes were first cycled at $60 \mathrm{~Hz}$ for $\sim 40$ min

637 and then at $10 \mathrm{~Hz}$ for at least 20 min until background current equilibration and throughout the

638 opto-ICSS behavioral session. Mice received a series of 3 experimenter-delivered stimulations

639 before and after the session to validate electrode functionality the day of each recording and for

640 generating voltammetric training sets (see Statistical Analyses). The opto-ICSS session began

641 at least $5 \mathrm{~min}$ after the final pre-session stimulation. The first half of each FSCV session

642 consisted of a standard opto-ICSS phase (CRF for the CRF cohort, LR sequence task for the

643 LR cohort) that was identical to the previous behavioral training sessions. At the conclusion of

644 this active Self-Stimulation phase, the both levers retracted and the house light turned off for a 5

645 min interim period, followed by a Passive Playback phase in which mice received non-

646 contingent stimulations with the same timing and stimulation parameters ( $1 \mathrm{~s} \mathrm{at} 50 \mathrm{~Hz})$ as in the

647 active Self-Stimulation phase. The timing of these non-contingent Passive Playback stimulations

648 was matched to the time stamps of stimulations earned during a given animal's preceding Self-

649 Stimulation phase, again ensuring that the stimulation rate and distribution of inter-stimulation

650 intervals were identical across both the active and passive phases for a given animal. Mice in

651 the LR sequence cohort also performed another $30 \mathrm{~min}$ of active LR sequence opto-ICSS

652 following the Passive Playback phase to permit assessment of possible temporal order effects

653 (Fig S3H-I). 
655 Mice also underwent additional FSCV recordings in several types of probe sessions, including 656 Omission, Delay, and Magnitude Probes for the CRF cohort, and Left and Right Lever Probes

657 for the LR cohort. These FSCV sessions consisted of the same basic protocol described above, 658 with active Self-Stimulation and non-contingent Passive Playback yoked within-subject. In

659 Omission Probe sessions, $20 \%$ of presses on the typically Active lever did not yield stimulation, 660 and instead caused a 5-s timeout period during which no further stimulation could be earned.

661 This timeout period was not explicitly cued with any overt stimulus, other than the absence of 662 the typical stimulation delivery. In Delay Probe sessions, 20\% of presses on the Active lever 663 resulted in stimulation that was delayed by $5 \mathrm{~s}$. As for the Omission Probe timeout period, no 664 further stimulation could be earned during this delay period. In Magnitude Probe sessions, 20\% 665 of Active lever presses yielded an increased magnitude of stimulation ( $5 \mathrm{~s}$ at $50 \mathrm{~Hz}$ ). For the LR 666 sequence cohort, the single-press probe sessions consisted of probe stimulations delivered on 667 a random subset of first lever presses after previous reinforcement, in addition to continuous 668 reinforcement for LR sequences as usual. For the Left Probe session, the next left lever press 669 following the last reinforcement was stimulated with $20 \%$ probability. Due to the lower probability 670 of an additional right lever press following a reinforcement, a right lever press following the last 671 reinforcement was stimulated with 50\% probability to collect enough probes for data analyses in 672 the Right Probe session. Probe sessions were recorded at least 2 days apart, with standard 673 opto-ICSS behavioral training sessions performed on the intervening days to allow return to 674 baseline performance.

SNc dopamine neurons were recorded and identified as previously described (Jin \& Costa,

680 2010; Howard et al., 2017). Briefly, neural activity was recorded using the MAP system

681 (Plexon), and spike activities first were sorted online with a build-in algorithm. Only spikes with

682 stereotypical waveforms distinguishable from noise and high signal-to-noise ratio were saved for

683 further analysis. Behavioral training and recording sessions were conducted as described above

684 for the CRF cohort. After recording the opto-ICSS session with active Self-Stimulation and

685 Passive Playback phases, the recorded spikes were further isolated into individual units using

686 offline sorting software (Offline Sorter, Plexon). Each individual unit displayed a clear refractory

687 period in the inter-spike interval histogram, with no spikes during the refractory period (larger 
688 than $1.3 \mathrm{~ms}$ ). To identify laser-evoked responses, neuronal firing was aligned to stimulation

689 onset and averaged across stimulations in 1-ms bins, and baseline was defined by averaging

690 neuronal firing in the $1 \mathrm{~s}$ preceding stimulation onset. The latency to respond to stimulation was

691 defined as the as the time to significant firing rate increase, with a threshold defined as $>99 \%$ of

692 baseline activity ( 3 standard deviations). Only units with short response latency (<10 ms) from

693 stimulation onset and high correlation between spontaneous and laser-evoked spike waveforms

694 ( $r$ > 0.95) were considered cre-positive, optogenetically identified dopamine neurons (Jin \&

695 Costa, 2010; Howard et al., 2017).

\section{Histology}

Mice were anesthetized with ketamine (100 mg/kg, i.p.) and xylazine (10 mg/kg, i.p.), and the FSCV recording site was marked by passing a $70 \mu \mathrm{A}$ current through the electrode for $20 \mathrm{~s}$. Mice were transcardially perfused with $0.01 \mathrm{M}$ phosphate-buffered saline (PBS) and then $4 \%$ paraformaldehyde (PFA) in PBS. Brains were removed, post-fixed in PFA at $4^{\circ}$ for $24 \mathrm{hr}$, and then stored at $4^{\circ}$ in a solution of $30 \%$ sucrose in $0.1 \mathrm{M}$ phosphate buffer until ready for cryosectioning. Tissue was sectioned at $50 \mu \mathrm{m}$ thickness on a freezing microtome, and striatal and SNc sections were mounted onto glass slides and coverslipped with AquaPoly mounting media containing DAPI (1:1000). Some sections also were processed for immunohistochemistry as previously described (Smith, Klug, Ross, et al., 2016; Geddes et al., 2018). Briefly, sections were washed 3 times for 15 min each in tris-buffered saline (TBS), and incubated for $1 \mathrm{hr}$ in blocking solution containing 3\% normal horse serum and $0.25 \%$ Triton-X 100 in TBS. Tissue was incubated for $48 \mathrm{hr}$ in primary antibody against tyrosine hydroxylase (anti-TH, raised in

712 rabbit, 1:1000, Abcam) and green fluorescent protein (anti-GFP, raised in chicken, 1:1000,

713 Novus Biologicals) in this blocking solution at $4^{\circ}$, washed twice for 15 min in TBS and then for

$71430 \mathrm{~min}$ in the blocking solution, and then incubated for $3 \mathrm{hr}$ in secondary antibody (anti-Chicken

715 AlexaFluor 488 and anti-Rabbit Cy3, each 1:250, Jackson ImmunoResearch ) in blocking

716 solution. Finally, sections were washed 3 times for 15 min in TBS, mounted onto slides, and

717 coverslipped with DAPI mounting media as above. Sections were imaged on a Zeiss LSM 710

718 confocal microscope with 10x and 20x objectives. All included FSCV animals were confirmed to

719 have electrode placement in the dorsal striatum and fiber optics targeting the SNc. 


\section{Statistical Analyses}

724 FSCV data were low-pass filtered at $2 \mathrm{kHz}$, aligned to each lever press and/or stimulation onset,

725 and background-subtracted using the mean voltammetric current in the $1 \mathrm{~s}$ prior to each aligned

726 event of interest. Dopamine responses were isolated using chemometric principal component

727 analysis with training sets consisting of cyclic voltammograms for dopamine, $\mathrm{pH}$, and electrode

728 drift (Keithley et al., 2009; Keithley \& Wightman, 2011; Howard et al., 2017). Electrode-specific

729 training sets were used for each animal and represented additional inclusion criteria for a given

730 electrode, but similar results were obtained when reanalyzing data with a standardized training

731 set across animals (Rodeberg et al., 2017). Changes in dopamine concentration were estimated

732 based on average post-implantation electrode sensitivity (Clark, Sandberg, et al., 2010). No

733 formal power analysis was conducted prior to experiments, but sample sizes were comparable

734 to previous publications.

Mean changes in dopamine concentration summarized in bar graphs throughout the results analyzed time periods spanning $0.5-1.5 \mathrm{~s}$ following the aligned event onset. Analysis of the Magnitude Probes also included a late time point at 4.5-5.5 s after Probe onset, as did supplementary analysis of Omission Probes with versus without additional presses during the timeout period. For the LR sequence cohort, analysis of non-reinforced press pairs was restricted to pairs with short inter-press intervals (IPI $<5 \mathrm{~s}$ ), consistent with the short duration of

742 most LR sequences. Analysis of the non-reinforced single Left and Right lever presses was

743 restricted to the first press following previous reinforcement, to match the press that could

744 receive probe stimulation in the corresponding single-press probe sessions. Analysis of non-

745 reinforced Right lever presses and Right Probe stimulations was restricted to those where the

746 animal did not first approach the Left lever, as determined by examination of the video, to

747 ensure that the right presses analyzed were individual actions and not part of a LR sequence.

748 Statistical analyses of behavioral and FSCV data consisted of $t$ tests and repeated-measures

749 ANOVAs with post-hoc tests corrected for multiple comparisons as indicated throughout the

750 corresponding figure legends. Stimulation-evoked dopamine traces also were analyzed with

751 Difference Traces that digitally subtracted the Passive Playback response from the Self-

752 Stimulation response for each pair of matched stimulations. Dopamine trace time courses

753 following event onset were analyzed with permutation tests (10,000 random shuffles) with a

754 cluster-based correction for multiple comparisons over time (Nichols \& Holmes, 2002; Maris \&

755 Oostenveld, 2007). For electrophysiological data analysis, neuronal firing was aligned to 
756 stimulation onset, averaged within each session phase, and smoothed with a Gaussian filter

757 (window size $=50 \mathrm{~ms}$, standard deviation $=10$ ) to construct peri-event time histograms for Self-

758 Stimulation and Passive Playback responses. Statistical analyses were performed in Prism

759 (GraphPad) and Matlab (MathWorks).

\section{ACKNOWLEDGMENTS}

763 We thank Jared Smith, Jason Klug, Sho Aoki, Roy Kim, Kanchi Mehta, Anthony Balolong-

764 Reyes, and Scott Ng-Evans for helpful discussions and technical assistance. This work was

765 supported by NIH grants K99MH119312 (N.G.H.) and R01NS083815 (X.J.), the Salk Institute

766 Pioneer Postdoctoral Endowment Fund and the Jonas Salk Fellowship (N.G.H.), and the

767 McKnight Neurobiology of Brain Disorders Award (X.J.).

\section{ADDITIONAL INFORMATION}

\section{$770 \quad$ FUNDING}

\begin{tabular}{|l|l|l|}
\hline Funder & Grant reference number & Author \\
\hline National Institutes of Health & K99MH119312 & Nick G Hollon \\
\hline National Institutes of Health & R01NS083815 & Xin Jin \\
\hline $\begin{array}{l}\text { Salk Institute Pioneer } \\
\text { Postdoctoral Endwoment }\end{array}$ & & Nick G Hollon \\
\hline Jonas Salk Fellowship & & Nick G Hollon \\
\hline McKnight Foundation & $\begin{array}{l}\text { McKnight Neurobiology of } \\
\text { Brain Disorders Award }\end{array}$ & Xin Jin \\
\hline
\end{tabular}

771 The funders had no role in study design, data collection and interpretation, or the decision to

772 submit the work for publication.

\section{AUTHOR CONTRIBUTIONS}

775 Nick G Hollon, Conceptualization, Methodology, Software, Validation, Formal analysis,

776 Investigation, Data curation, Writing-original draft, Writing—review and editing, Visualization,

777 Supervision, Project administration, Funding acquisition; Elora W Williams, Validation, Formal

778 analysis, Investigation, Data curation, Writing—review and editing; Christopher D Howard,

779 Conceptualization, Methodology, Validation, Writing—review and editing, Supervision; 
780 Hao Li, Methodology, Software, Validation, Formal analysis, Investigation, Data curation,

781 Writing—review and editing, Visualization; Tavish I Traut, Validation, Investigation, Writing-

782 review and editing; Xin Jin, Conceptualization, Methodology, Validation, Resources, Writing-

783 original draft, Writing—review and editing, Visualization, Supervision, Project administration,

784 Funding acquisition

785

786 COMPETING INTERESTS

787 The authors declare no competing interests.

788 


\section{REFERENCES}

Balleine, B.W., 2019. The Meaning of Behavior: Discriminating Reflex and Volition in the Brain. Neuron 104, 47-62. doi:10.1016/j.neuron.2019.09.024

Barnes, T.D., Kubota, Y., Hu, D., Jin, D.Z., Graybiel, A.M., 2005. Activity of striatal neurons reflects dynamic encoding and recoding of procedural memories. Nature 437, 1158-1161. doi:10.1038/nature04053

Barter, J.W., Li, S., Lu, D., Bartholomew, R.A., Rossi, M.A., Shoemaker, C.T., Salas-Meza, D., Gaidis, E., Yin, H.H., 2015. Beyond reward prediction errors: the role of dopamine in movement kinematics. Front Integr Neurosci 9, 39. doi:10.3389/fnint.2015.00039

Belin, D., Everitt, B.J., 2008. Cocaine seeking habits depend upon dopamine-dependent serial connectivity linking the ventral with the dorsal striatum. Neuron 57, 432-441.

800 doi:10.1016/j.neuron.2007.12.019

Brazhnik, E., Shah, F., Tepper, J.M., 2008. GABAergic afferents activate both GABAA and GABAB receptors in mouse substantia nigra dopaminergic neurons in vivo. J Neurosci 28, 10386-10398. doi:10.1523/JNEUROSCI.2387-08.2008

Chang, C.Y., Esber, G.R., Marrero-Garcia, Y., Yau, H.-J., Bonci, A., Schoenbaum, G., 2016. Brief optogenetic inhibition of dopamine neurons mimics endogenous negative reward prediction errors. Nat Neurosci 19, 111-116. doi:10.1038/nn.4191

Clancy, K.B., Koralek, A.C., Costa, R.M., Feldman, D.E., Carmena, J.M., 2014. Volitional modulation of optically recorded calcium signals during neuroprosthetic learning. Nat Neurosci 17, 807-809. doi:10.1038/nn.3712

Clark, J.J., Sandberg, S.G., Wanat, M.J., Gan, J.O., Horne, E.A., Hart, A.S., Akers, C.A., Parker, J.G., Willuhn, I., Martinez, V., Evans, S.B., Stella, N., Phillips, P.E.M., 2010. Chronic microsensors for longitudinal, subsecond dopamine detection in behaving animals. Nat Methods 7, 126-129. doi:10.1038/nmeth.1412

Coddington, L.T., Dudman, J.T., 2018. The timing of action determines reward prediction signals in identified midbrain dopamine neurons. Nat Neurosci 21, 1563-1573. doi:10.1038/s41593-018-0245-7

Coddington, L.T., Dudman, J.T., 2019. Learning from Action: Reconsidering Movement Signaling in Midbrain Dopamine Neuron Activity. Neuron 104, 63-77. doi:10.1016/j.neuron.2019.08.036

Collins, A.L., Greenfield, V.Y., Bye, J.K., Linker, K.E., Wang, A.S., Wassum, K.M., 2016. Dynamic mesolimbic dopamine signaling during action sequence learning and expectation violation. Sci Rep 6, 20231. doi:10.1038/srep20231

Cohen, J.Y., Haesler, S., Vong, L., Lowell, B.B., Uchida, N., 2012. Neuron-type-specific signals for reward and punishment in the ventral tegmental area. Nature 482, 85-88. doi:10.1038/nature10754

Corbit, L.H., Janak, P.H., 2007. Inactivation of the lateral but not medial dorsal striatum eliminates the excitatory impact of Pavlovian stimuli on instrumental responding. J Neurosci 27, 13977-13981. doi:10.1523/JNEUROSCI.4097-07.2007

Covey, D.P., Cheer, J.F., 2019. Accumbal Dopamine Release Tracks the Expectation of Dopamine Neuron-Mediated Reinforcement. CellReports 27, 481-490.e3. doi:10.1016/j.celrep.2019.03.055

Crapse, T.B., Sommer, M.A., 2008. Corollary discharge across the animal kingdom. Nat Rev Neurosci 9, 587-600. doi:10.1038/nrn2457 
834

835

836

837

838

839

840

841

842

843

844

845

846

847

848

849

850

851

852

853

854

855

856

857

858

859

860

861

862

863

864

865

866

867

868

869

870

871

872

873

874

875

876

877

da Silva, J.A., Tecuapetla, F., Paixão, V., Costa, R.M., 2018. Dopamine neuron activity before action initiation gates and invigorates future movements. Nature 554, 244-248. doi:10.1038/nature25457

de Jong, J.W., Afjei, S.A., Dorocic, I.P., Peck, J.R., Liu, C., Kim, C.K., Tian, L., Deisseroth, K., Lammel, S., 2018. A Neural Circuit Mechanism for Encoding Aversive Stimuli in the Mesolimbic Dopamine System. Neuron 1-42. doi:10.1016/j.neuron.2018.11.005

Dodson, P.D., Dreyer, J.K., Jennings, K.A., Syed, E.C.J., Wade-Martins, R., Cragg, S.J., Bolam, J.P., Magill, P.J., 2016. Representation of spontaneous movement by dopaminergic neurons is cell-type selective and disrupted in parkinsonism. Proc Natl Acad Sci USA 113, E2180-8. doi:10.1073/pnas.1515941113

Engelhard, B., Finkelstein, J., Cox, J., Fleming, W., Jang, H.J., Ornelas, S., Koay, S.A., Thiberge, S.Y., Daw, N.D., Tank, D.W., Witten, I.B., 2019. Specialized coding of sensory, motor and cognitive variables in VTA dopamine neurons. Nature 570, 509-513. doi:10.1038/s41586-019-1261-9

Eshel, N., Bukwich, M., Rao, V., Hemmelder, V., Tian, J., Uchida, N., 2015. Arithmetic and local circuitry underlying dopamine prediction errors. Nature 525, 243-246. doi:10.1038/nature14855

Eshel, N., Tian, J., Bukwich, M., Uchida, N., 2016. Dopamine neurons share common response function for reward prediction error. Nat Neurosci 19, 479-486. doi:10.1038/nn.4239

Evans, R.C., Twedell, E.L., Zhu, M., Ascencio, J., Zhang, R., Khaliq, Z.M., 2020. Functional Dissection of Basal Ganglia Inhibitory Inputs onto Substantia Nigra Dopaminergic Neurons. CellReports 32, 108156. doi:10.1016/j.celrep.2020.108156

Faure, A., Haberland, U., Condé, F., Massioui, El, N., 2005. Lesion to the nigrostriatal dopamine system disrupts stimulus-response habit formation. Journal of Neuroscience 25, 2771-2780. doi:10.1523/JNEUROSCI.3894-04.2005

Fee, M.S., 2014. The role of efference copy in striatal learning. Curr Opin Neurobiol 25, 194200. doi:10.1016/j.conb.2014.01.012

Fiorillo, C.D., Tobler, P.N., Schultz, W., 2003. Discrete coding of reward probability and uncertainty by dopamine neurons. Science 299, 1898-1902. doi:10.1126/science.1077349

Flagel, S.B., Clark, J.J., Robinson, T.E., Mayo, L., Czuj, A., Willuhn, I., Akers, C.A., Clinton, S.M., Phillips, P.E.M., Akil, H., 2011. A selective role for dopamine in stimulus-reward learning. Nature 469, 53-57. doi:10.1038/nature09588

Gadagkar, V., Puzerey, P.A., Chen, R., Baird-Daniel, E., Farhang, A.R., Goldberg, J.H., 2016. Dopamine neurons encode performance error in singing birds. Science 354, 1278-1282. doi:10.1126/science.aah6837

Gallistel, C.R. (1980). The Organization of Action: A New Synthesis. (Hillsdale, NJ: Lawrence Erlbaum Associates).

Garris, P.A., Kilpatrick, M., Bunin, M.A., Michael, D., Walker, Q.D., Wightman, R.M., 1999. Dissociation of dopamine release in the nucleus accumbens from intracranial selfstimulation. Nature 398, 67-69. doi:10.1038/18019

Geddes, C.E., Li, H., Jin, X., 2018. Optogenetic Editing Reveals the Hierarchical Organization of Learned Action Sequences. Cell 174, 32-43.e15. doi:10.1016/j.cell.2018.06.012

Graybiel, A.M., 1998. The basal ganglia and chunking of action repertoires. Neurobiol Learn Mem 70, 119-136. doi:10.1006/nIme.1998.3843 
878

879

880

881

882

883

884

885

886

887

888

889

890

891

892

893

894

895

896

897

898

899

900

901

902

903

904

905

906

907

908

909

910

911

912

913

914

915

916

917

918

919

920

921

922

Gremel, C.M., Costa, R.M., 2013. Orbitofrontal and striatal circuits dynamically encode the shift between goal-directed and habitual actions. Nature Communications 4, 2264. doi:10.1038/ncomms3264

Hamid, A.A., Pettibone, J.R., Mabrouk, O.S., Hetrick, V.L., Schmidt, R., Vander Weele, C.M., Kennedy, R.T., Aragona, B.J., Berke, J.D., 2016. Mesolimbic dopamine signals the value of work. Nat Neurosci 19, 117-126. doi:10.1038/nn.4173

Hart, A.S., Rutledge, R.B., Glimcher, P.W., Phillips, P.E.M., 2014. Phasic dopamine release in the rat nucleus accumbens symmetrically encodes a reward prediction error term. $\mathrm{J}$ Neurosci 34, 698-704. doi:10.1523/JNEUROSCI.2489-13.2014

Hikosaka, O., Miyashita, K., Miyachi, S., Sakai, K., Lu, X., 1998. Differential roles of the frontal cortex, basal ganglia, and cerebellum in visuomotor sequence learning. Neurobiol Learn Mem 70, 137-149. doi:10.1006/nlme.1998.3844

Hollon, N.G., Arnold, M.M., Gan, J.O., Walton, M.E., Phillips, P.E.M., 2014. Dopamineassociated cached values are not sufficient as the basis for action selection. Proc Natl Acad Sci USA 111, 18357-18362. doi:10.1073/pnas.1419770111

Hong, S., Hikosaka, O., 2008. The globus pallidus sends reward-related signals to the lateral habenula. Neuron 60, 720-729. doi:10.1016/j.neuron.2008.09.035

Houk, J.C., Adams, J.L., Barto, A.G., 1995. A model of how the basal ganglia generate and use neural signals that predict reinforcement. Models of Information Processing in the Basal Ganglia, 249-270.

Howard, C.D., Li, H., Geddes, C.E., Jin, X., 2017. Dynamic Nigrostriatal Dopamine Biases Action Selection. Neuron 93, 1436-1450.e8. doi:10.1016/j.neuron.2017.02.029

Howe, M.W., Dombeck, D.A., 2016. Rapid signalling in distinct dopaminergic axons during locomotion and reward. Nature 535, 505-510. doi:10.1038/nature18942

Ilango, A., Kesner, A.J., Keller, K.L., Stuber, G.D., Bonci, A., Ikemoto, S., 2014. Similar roles of substantia nigra and ventral tegmental dopamine neurons in reward and aversion. $J$ Neurosci 34, 817-822. doi:10.1523/JNEUROSCI.1703-13.2014

Jin, X., Costa, R.M., 2010. Start/stop signals emerge in nigrostriatal circuits during sequence learning. Nature 466, 457-462. doi:10.1038/nature09263

Jin, X., Costa, R.M., 2015. Shaping action sequences in basal ganglia circuits. Curr Opin Neurobiol 33, 188-196. doi:10.1016/j.conb.2015.06.011

Jin, X., Tecuapetla, F., Costa, R.M., 2014. Basal ganglia subcircuits distinctively encode the parsing and concatenation of action sequences. Nat Neurosci 17, 423-430. doi:10.1038/nn.3632

Keiflin, R., Pribut, H.J., Shah, N.B., Janak, P.H., 2019. Ventral Tegmental Dopamine Neurons Participate in Reward Identity Predictions. Curr Biol 29, 93-103.e3. doi:10.1016/j.cub.2018.11.050

Keithley, R.B., Heien, M.L., Wightman, R.M., 2009. Multivariate concentration determination using principal component regression with residual analysis. Trends in Analytical Chemistry 28, 1127-1136. doi:10.1016/j.trac.2009.07.002

Keithley, R.B., Wightman, R.M., 2011. Assessing principal component regression prediction of neurochemicals detected with fast-scan cyclic voltammetry. ACS Chem Neurosci 2, 514525. doi:10.1021/cn200035u

Keller, G.B., Mrsic-Flogel, T.D., 2018. Predictive Processing: A Canonical Cortical Computation. Neuron 100, 424-435. doi:10.1016/j.neuron.2018.10.003 
923

924

925

926

927

928

929

930

931

932

933

934

935

936

937

938

939

940

941

942

943

944

945

946

947

948

949

950

951

952

953

954

955

956

957

958

959

960

961

962

963

964

965

966

967

Kilpatrick, M.R., Rooney, M.B., Michael, D.J., Wightman, R.M., 2000. Extracellular dopamine dynamics in rat caudate-putamen during experimenter-delivered and intracranial selfstimulation. Neuroscience 96, 697-706. doi:10.1016/s0306-4522(99)00578-3

Kim, H.F., Amita, H., Hikosaka, O., 2017. Indirect Pathway of Caudal Basal Ganglia for Rejection of Valueless Visual Objects. Neuron 94, 920-930.e3. doi:10.1016/j.neuron.2017.04.033

Kim, H.F., Ghazizadeh, A., Hikosaka, O., 2015. Dopamine Neurons Encoding Long-Term Memory of Object Value for Habitual Behavior. Cell 163, 1165-1175. doi:10.1016/j.cell.2015.10.063

Klaus, A., Alves da Silva, J., Costa, R.M., 2019. What, If, and When to Move: Basal Ganglia Circuits and Self-Paced Action Initiation. Annu Rev Neurosci 42, 459-483. doi:10.1146/annurev-neuro-072116-031033

Knowlton, B.J., Mangels, J.A., Squire, L.R., 1996. A neostriatal habit learning system in humans. Science 273, 1399-1402. doi:10.1126/science.273.5280.1399

Koralek, A.C., Jin, X., Long, J.D., Costa, R.M., Carmena, J.M., 2012. Corticostriatal plasticity is necessary for learning intentional neuroprosthetic skills. Nature 483, 331-335. doi:10.1038/nature10845

Kruse, J.M., Overmier, J.B., Konz, W.A., Rokke, E., 1983. Pavlovian conditioned stimulus effects upon instrumental choice behavior are reinforcer specific. Learning and Motivation $14,165-181$.

Lashley, K.S. (1951). The problem of serial order in behavior. In Cerebral Mechanisms in Behavior: The Hixon Symposium, L.A. Jeffress, ed., (New York: Wiley), pp. 112-136.

Lau, B., Glimcher, P.W., 2008. Value representations in the primate striatum during matching behavior. Neuron 58, 451-463. doi:10.1016/j.neuron.2008.02.021

Lerner, T.N., Shilyansky, C., Davidson, T.J., Evans, K.E., Beier, K.T., Zalocusky, K.A., Crow, A.K., Malenka, R.C., Luo, L., Tomer, R., Deisseroth, K., 2015. Intact-brain analyses reveal distinct information carried by SNc dopamine subcircuits. Cell 162, 635-647. doi:10.1016/j.cell.2015.07.014

Madisen, L., Mao, T., Koch, H., Zhuo, J.-M., Berenyi, A., Fujisawa, S., Hsu, Y.-W.A., Garcia, A.J., Gu, X., Zanella, S., Kidney, J., Gu, H., Mao, Y., Hooks, B.M., Boyden, E.S., Buzsáki, G., Ramirez, J.-M., Jones, A.R., Svoboda, K., Han, X., Turner, E.E., Zeng, H., 2012. A toolbox of Cre-dependent optogenetic transgenic mice for light-induced activation and silencing. Nat Neurosci 15, 793-802. doi:10.1038/nn.3078

Maes, E.J.P., Sharpe, M.J., Usypchuk, A.A., Lozzi, M., Chang, C.Y., Gardner, M.P.H., Schoenbaum, G., lordanova, M.D., 2020. Causal evidence supporting the proposal that dopamine transients function as temporal difference prediction errors. Nat Neurosci 108, 15647. doi:10.1038/s41593-019-0574-1

Maris, E., Oostenveld, R., 2007. Nonparametric statistical testing of EEG- and MEG-data. J Neurosci Methods 164, 177-190. doi:10.1016/j.jneumeth.2007.03.024

Matamales, M., McGovern, A.E., Mi, J.D., Mazzone, S.B., Balleine, B.W., Bertran-Gonzalez, J., 2020. Local D2- to D1-neuron transmodulation updates goal-directed learning in the striatum. Science 367, 549-555. doi:10.1126/science.aaz5751

Matsumoto, N., Hanakawa, T., Maki, S., Graybiel, A.M., Kimura, M., 1999. Role of nigrostriatal dopamine system in learning to perform sequential motor tasks in a predictive manner. J Neurophysiol 82, 978-998. doi:10.1152/jn.1999.82.2.978 
968

969

970

971

972

973

974

975

976

977

978

979

980

981

982

983

984

985

986

987

988

989

990

991

992

993

994

995

996

997

998

999

1000

1001

1002

1003

1004

1005

1006

1007

1008

1009

1010

1011

Matsumoto, M., Hikosaka, O., 2009. Two types of dopamine neuron distinctly convey positive and negative motivational signals. Nature 459, 837-841. doi:10.1038/nature08028

Menegas, W., Akiti, K., Amo, R., Uchida, N., Watabe-Uchida, M., 2018. Dopamine neurons projecting to the posterior striatum reinforce avoidance of threatening stimuli. Nat Neurosci 21, 1421-1430. doi:10.1038/s41593-018-0222-1

Menegas, W., Bergan, J.F., Ogawa, S.K., Isogai, Y., Umadevi Venkataraju, K., Osten, P., Uchida, N., Watabe-Uchida, M., 2015. Dopamine neurons projecting to the posterior striatum form an anatomically distinct subclass. eLife 4, e10032. doi:10.7554/eLife.10032

Montague, P.R., Dayan, P., Sejnowski, T.J., 1996. A framework for mesencephalic dopamine systems based on predictive Hebbian learning. J Neurosci 16, 1936-1947.

Morris, G., Arkadir, D., Nevet, A., Vaadia, E., Bergman, H., 2004. Coincident but distinct messages of midbrain dopamine and striatal tonically active neurons. Neuron 43, 133-143. doi:10.1016/j.neuron.2004.06.012

Neely, R.M., Koralek, A.C., Athalye, V.R., Costa, R.M., Carmena, J.M., 2018. Volitional Modulation of Primary Visual Cortex Activity Requires the Basal Ganglia. Neuron 97, 13561368.e4. doi:10.1016/j.neuron.2018.01.051

Nichols, T.E., Holmes, A.P., 2002. Nonparametric permutation tests for functional neuroimaging: a primer with examples. Hum Brain Mapp 15, 1-25.

Owesson-White, C.A., Cheer, J.F., Beyene, M., Carelli, R.M., Mark Wightman, R., 2008. Dynamic changes in accumbens dopamine correlate with learning during intracranial selfstimulation. Proc Natl Acad Sci USA 105, 11957-11962. doi:10.1073/pnas.0803896105

Panigrahi, B., Martin, K.A., Li, Y., Graves, A.R., Vollmer, A., Olson, L., Mensh, B.D., Karpova, A.Y., Dudman, J.T., 2015. Dopamine Is Required for the Neural Representation and Control of Movement Vigor. Cell 162, 1418-1430. doi:10.1016/j.cell.2015.08.014

Parker, N.F., Cameron, C.M., Taliaferro, J.P., Lee, J., Choi, J.Y., Davidson, T.J., Daw, N.D., Witten, I.B., 2016. Reward and choice encoding in terminals of midbrain dopamine neurons depends on striatal target. Nat Neurosci 19, 845-854. doi:10.1038/nn.4287

Rao, R.P., Ballard, D.H., 1999. Predictive coding in the visual cortex: a functional interpretation of some extra-classical receptive-field effects. Nat Neurosci 2, 79-87. doi:10.1038/4580

Redgrave, P., Gurney, K., 2006. The short-latency dopamine signal: a role in discovering novel actions? Nat Rev Neurosci 7, 967-975. doi:10.1038/nrn2022

Rodeberg, N.T., Johnson, J.A., Bucher, E.S., Wightman, R.M., 2016. Dopamine Dynamics during Continuous Intracranial Self-Stimulation: Effect of Waveform on Fast-Scan Cyclic Voltammetry Data. ACS Chem Neurosci 7, 1508-1518. doi:10.1021/acschemneuro.6b00142

Rodeberg, N.T., Sandberg, S.G., Johnson, J.A., Phillips, P.E.M., Wightman, R.M., 2017. Hitchhiker's Guide to Voltammetry: Acute and Chronic Electrodes for in Vivo Fast-Scan Cyclic Voltammetry. ACS Chem Neurosci 8, 221-234. doi:10.1021/acschemneuro.6b00393

Roesch, M.R., Calu, D.J., Schoenbaum, G., 2007. Dopamine neurons encode the better option in rats deciding between differently delayed or sized rewards. Nat Neurosci 10, 1615-1624. doi:10.1038/nn2013

Roesch, M.R., Singh, T., Leon Brown, P., Mullins, S.E., Schoenbaum, G., 2009. Ventral striatal neurons encode the value of the chosen action in rats deciding between differently delayed or sized rewards. J Neurosci 29, 13365-13376. doi:10.1523/JNEUROSCI.2572-09.2009 
1012

1013

1014

1015

1016

1017

1018

1019

1020

1021

1022

1023

1024

1025

1026

1027

1028

1029

1030

1031

1032

1033

1034

1035

1036

1037

1038

1039

1040

1041

1042

1043

1044

1045

1046

1047

1048

1049

1050

1051

1052

1053

1054

1055

Rossi, M.A., Sukharnikova, T., Hayrapetyan, V.Y., Yang, L., Yin, H.H., 2013. Operant selfstimulation of dopamine neurons in the substantia nigra. PLoS ONE 8, e65799. doi:10.1371/journal.pone.0065799

Samejima, K., Ueda, Y., Doya, K., Kimura, M., 2005. Representation of action-specific reward values in the striatum. Science 310, 1337-1340. doi:10.1126/science.1115270

Saunders, B.T., Richard, J.M., Margolis, E.B., Janak, P.H., 2018. Dopamine neurons create Pavlovian conditioned stimuli with circuit-defined motivational properties. Nat Neurosci 1-17. doi:10.1038/s41593-018-0191-4

Schneider, D.M., Sundararajan, J., Mooney, R., 2018. A cortical filter that learns to suppress the acoustic consequences of movement. Nature 561, 391-395. doi:10.1038/s41586-018-05205

Schultz, W., Dayan, P., Montague, P.R., 1997. A neural substrate of prediction and reward. Science 275, 1593-1599. doi:10.1126/science.275.5306.1593

Smith, J.B., Klug, J.R., Ross, D.L., Howard, C.D., Hollon, N.G., Ko, V.I., Hoffman, H., Callaway, E.M., Gerfen, C.R., Jin, X., 2016. Genetic-Based Dissection Unveils the Inputs and Outputs of Striatal Patch and Matrix Compartments. Neuron 91, 1069-1084. doi:10.1016/j.neuron.2016.07.046

Sparta, D.R., Stamatakis, A.M., Phillips, J.L., Hovelsø, N., van Zessen, R., Stuber, G.D., 2011. Construction of implantable optical fibers for long-term optogenetic manipulation of neural circuits. Nat Protoc 7, 12-23. doi:10.1038/nprot.2011.413

Steinberg, E.E., Gore, F., Heifets, B.D., Taylor, M.D., Norville, Z.C., Beier, K.T., Földy, C., Lerner, T.N., Luo, L., Deisseroth, K., Malenka, R.C., 2020. Amygdala-Midbrain Connections Modulate Appetitive and Aversive Learning. Neuron. doi:10.1016/j.neuron.2020.03.016

Steinberg, E.E., Keiflin, R., Boivin, J.R., Witten, I.B., Deisseroth, K., Janak, P.H., 2013. A causal link between prediction errors, dopamine neurons and learning. Nat Neurosci 16, 966-973. doi:10.1038/nn.3413

Sutton, R.S., Barto, A.G., 2018. Reinforcement learning: An introduction. (Cambridge,MA: MIT Press).

Tachibana, Y., Hikosaka, O., 2012. The primate ventral pallidum encodes expected reward value and regulates motor action. Neuron 76, 826-837. doi:10.1016/j.neuron.2012.09.030

Takahashi, Y.K., Batchelor, H.M., Liu, B., Khanna, A., Morales, M., Schoenbaum, G., 2017. Dopamine Neurons Respond to Errors in the Prediction of Sensory Features of Expected Rewards. Neuron 95, 1395-1405.e3. doi:10.1016/j.neuron.2017.08.025

Tepper, J.M., Lee, C.R., 2007. GABAergic control of substantia nigra dopaminergic neurons. Prog Brain Res 160, 189-208. doi:10.1016/S0079-6123(06)60011-3

Tsutsui-Kimura, I., Matsumoto, H., Akiti, K., Yamada, M.M., Uchida, N., Watabe-Uchida, M., 2020. Distinct temporal difference error signals in dopamine axons in three regions of the striatum in a decision-making task. eLife 9, 3327. doi:10.7554/eLife.62390

Wang, L.P., Li, F., Wang, D., Xie, K., Wang, D., Shen, X., Tsien, J.Z., 2011. NMDA Receptors in Dopaminergic Neurons Are Crucial for Habit Learning. Neuron 72, 1055-1066. doi:10.1016/j.neuron.2011.10.019

Wassum, K.M., Ostlund, S.B., Maidment, N.T., 2012. Phasic mesolimbic dopamine signaling precedes and predicts performance of a self-initiated action sequence task. Biol Psychiatry 71, 846-854. doi:10.1016/j.biopsych.2011.12.019 
1056

1057

1058

1059

1060

1061

1062

1063

1064

1065

1066

1067

1068

1069

1070

1071

1072

1073

1074

1075

1076

1077

1078
Watabe-Uchida, M., Zhu, L., Ogawa, S.K., Vamanrao, A., Uchida, N., 2012. Whole-brain mapping of direct inputs to midbrain dopamine neurons. Neuron $74,858-873$. doi:10.1016/j.neuron.2012.03.017

Wise, R.A., 2002. Brain reward circuitry: insights from unsensed incentives. Neuron 36, 229240. doi:10.1016/s0896-6273(02)00965-0

Witten, I.B., Steinberg, E.E., Lee, S.Y., Davidson, T.J., Zalocusky, K.A., Brodsky, M., Yizhar, O., Cho, S.L., Gong, S., Ramakrishnan, C., Stuber, G.D., Tye, K.M., Janak, P.H., Deisseroth, K., 2011. Recombinase-driver rat lines: tools, techniques, and optogenetic application to dopamine-mediated reinforcement. Neuron 72, 721-733. doi:10.1016/j.neuron.2011.10.028

Wolpert, D.M., Ghahramani, Z., Jordan, M.I., 1995. An internal model for sensorimotor integration. Science 269, 1880-1882. doi:10.1126/science.7569931

Wurtz, R.H., 2018. Corollary Discharge Contributions to Perceptual Continuity Across Saccades. Annu. Rev. Vis. Sci. 4, 215-237. doi:10.1146/annurev-vision-102016-061207

Yin, H.H., Ostlund, S.B., Balleine, B.W., 2008. Reward-guided learning beyond dopamine in the nucleus accumbens: the integrative functions of cortico-basal ganglia networks. Eur $\mathrm{J}$ Neurosci 28, 1437-1448. doi:10.1111/j.1460-9568.2008.06422.x

Yin, H.H., Ostlund, S.B., Knowlton, B.J., Balleine, B.W., 2005. The role of the dorsomedial striatum in instrumental conditioning. Eur J Neurosci 22, 513-523. doi:10.1111/j.14609568.2005.04218.x

Zhuang, X., Masson, J., Gingrich, J.A., Rayport, S., Hen, R., 2005. Targeted gene expression in dopamine and serotonin neurons of the mouse brain. J Neurosci Methods 143, 27-32. doi:10.1016/j.jneumeth.2004.09.020 
A

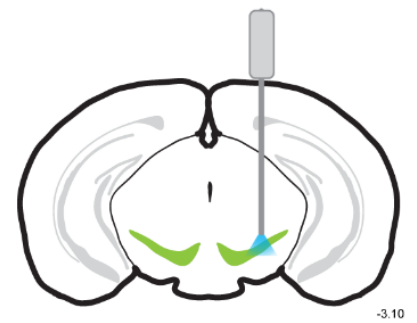

$\mathrm{D}$

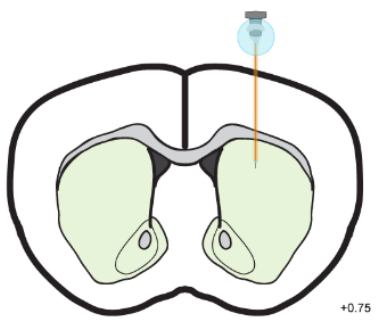

G

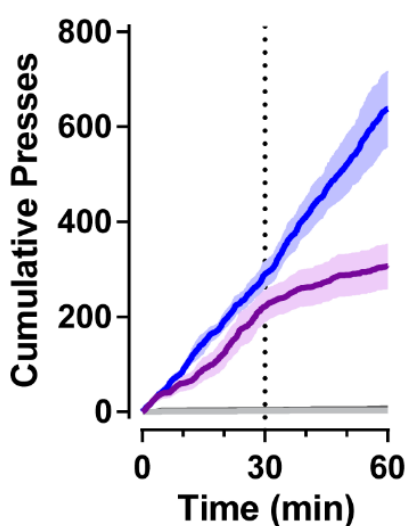

Previous opto-ICSS Contingency

Session:

- Active Lever

Degradation:

- Active Lever

E

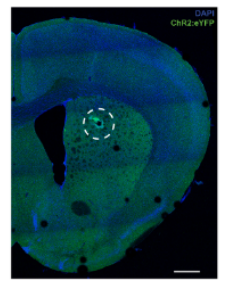

C

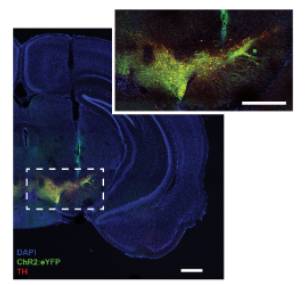

F
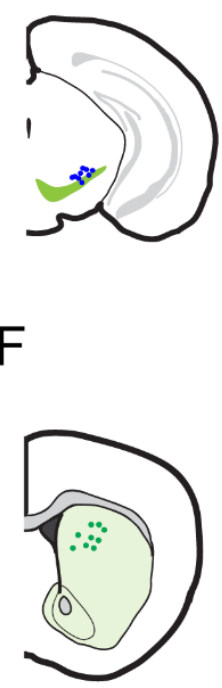

$\mathrm{H}$

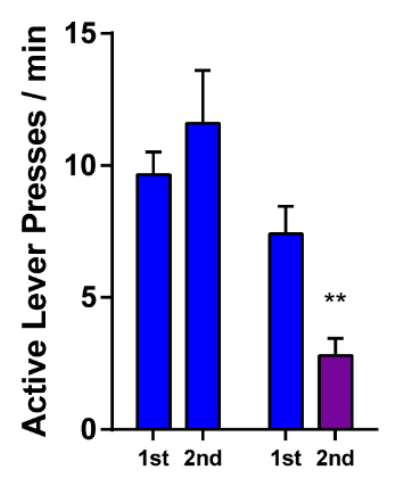

Half of Session

Previous $30 \mathrm{~min}$ opto-ICSS, opto-ICSS
Session
I

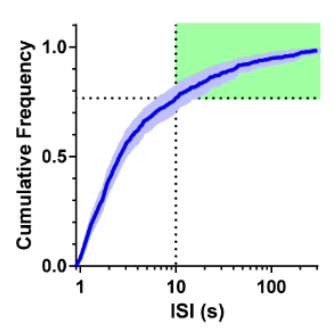

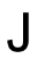

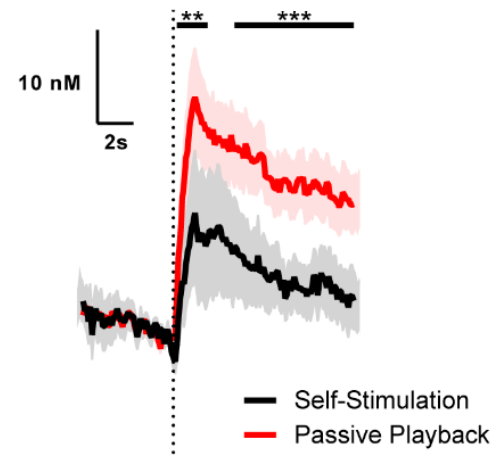

K

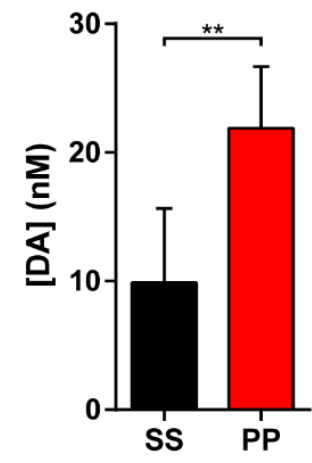


1081

1082

1083

1084

1085

1086

1087

1088

1089

1090

1091

1092

1093

1094

1095

1096

1097

1098

1099

1100

1101

1102

1103

1104

1105

1106

1107

1108

1109
Figure 1-figure supplement 1. Opto-ICSS CRF Cohort Histology, Contingency Degradation, and Bout Initiations. (A) Coronal schematic of fiber optic placement targeting the SNc (-3.10 mm posterior from bregma). (B) Representative image of fiber optic placement over the SNc in animal selectively expressing ChR2-eYFP in TH-positive dopamine neurons (scale bars $=500 \mu \mathrm{m})$. (C) Fiber optic placement for mice in the CRF cohort. (D) Coronal schematic of FSCV carbon-fiber microelectrode placement in the dorsal striatum $(+0.75 \mathrm{~mm}$ anterior to bregma). (E) Representative image of FSCV carbon-fiber microelectrode placement in the dorsal striatum (scale bar $=500 \mu \mathrm{m}$ ). The lesion was made by passing a current through the electrode just before perfusion after the conclusion of all experiments (see Methods). (F) FSCV electrode placement for mice in the CRF cohort. (G) Cumulative presses over time within the contingency degradation test session (30 min opto-ICSS followed by the 30 min contingency degradation test phase), overlaid with performance throughout the previous day's standard opto-ICSS session for comparison ( $n=6$ mice). (H) Summary of mean Active lever press rate during each phase of the contingency degradation test session (as in Fig. 1A), compared to the preceding day's standard opto-ICSS session (two-way repeated-measures ANOVA: main effect of Day, $F_{1,5}=8.157, P=0.0356$; Day by Half of Session interaction, $F_{1,5}=25.30, P=0.0040$; Sidak's multiple comparisons tests: Contingency Degradation $1^{\text {st }}$ vs. $2^{\text {nd }}$ Half, $P=0.0082 ; 2^{\text {nd }}$ Half of Previous Day vs. Contingency Degradation test phase, $P=0.0004$ ). (I) Cumulative frequency distribution of inter-stimulation intervals (ISIs) from the opto-ICSS FSCV recording session ( $n=9$ mice). Green shading indicates ISIs $>10 \mathrm{~s}$, used to define bout initiation for the subset of stimulations analyzed in $(\mathrm{J}-\mathrm{K})$. (J) Mean dopamine concentration change to boutinitiating Self-Stimulation (ISI > 10s since previous stimulation) and corresponding Passive Playback stimulations. Black bars indicate time points where the Self-Stimulation response significantly differs from Passive Playback (permutation test, $P S=0.007$ and 0.0001 for first and second time clusters, respectively). (K) Mean change in dopamine concentration for the boutinitiating subset of stimulations in $(\mathrm{J}) .\left(t_{8}=3.600, P=0.0070\right)$. Ctg. Deg., Contingency Degradation; ISI, Inter-stimulation interval; SS, Self-Stimulation; PP, Passive Playback. Error bars are SEM here and for below figures. 
bioRxiv preprint doi: https://doi.org/10.1101/2021.01.25.428032; this version posted January 26, 2021. The copyright holder for this preprint (which was not certified by peer review) is the author/funder, who has granted bioRxiv a license to display the preprint in perpetuity. It is made available under aCC-BY-NC-ND 4.0 International license.

A

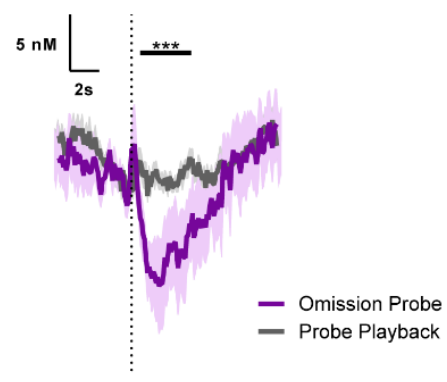

C

No Press during Stim With Press during Stim

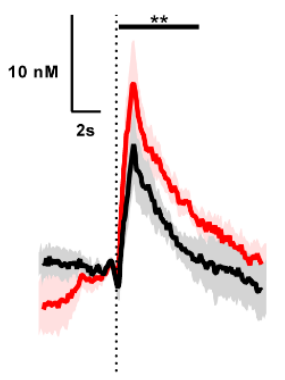

$\mathrm{E}$

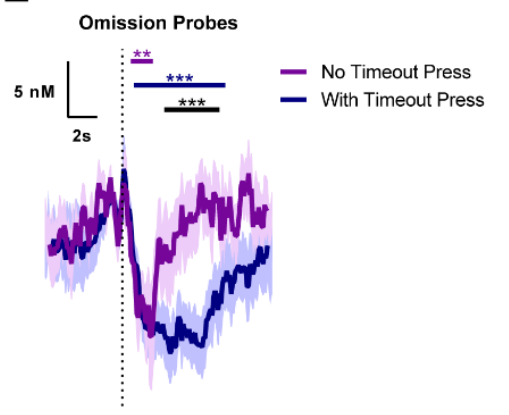

$\mathrm{F}$
B

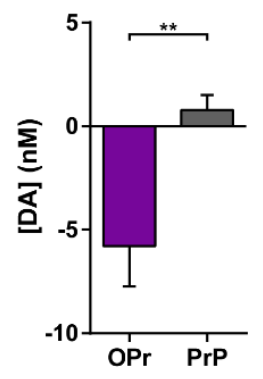

$\mathrm{D}$

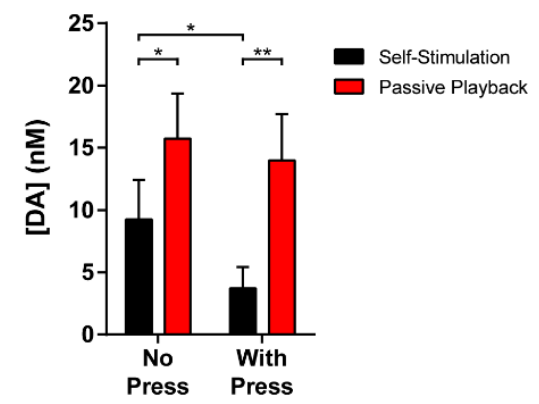

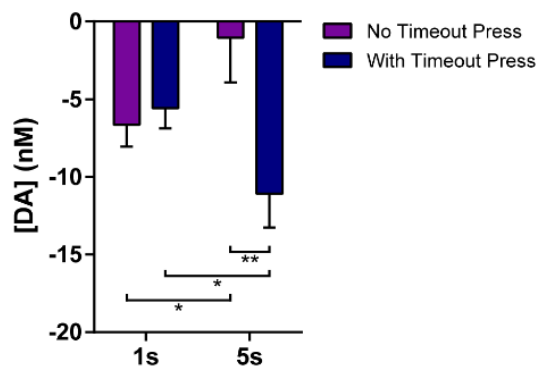

G

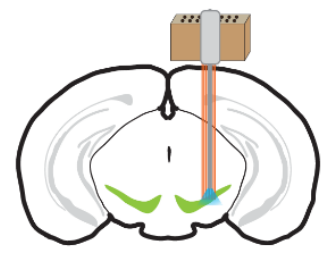

$\mathrm{H}$<smiles>C#CCC</smiles>

I
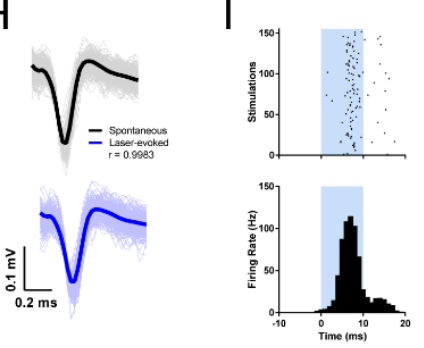

$\mathrm{J}$ selt:stimulation $\mathrm{K}$

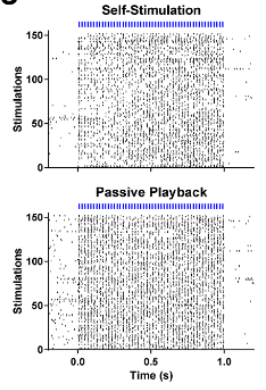

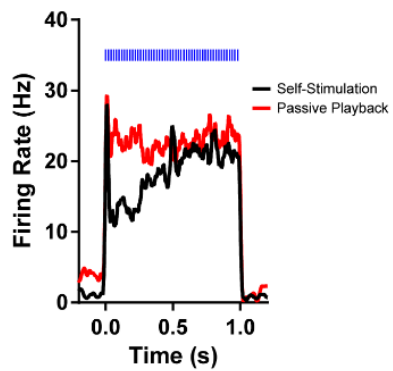

L

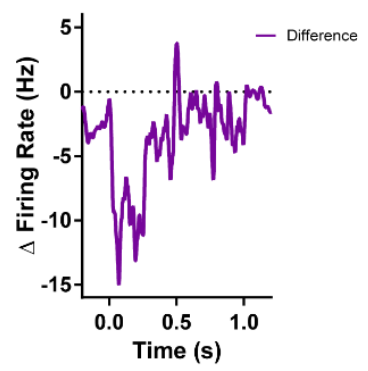


1111 Figure 2-figure supplement 1. Inhibition of Dopamine to Isolated Omissions, Augmented

1112 Suppression by Additional Presses, and In Vivo Electrophysiology. (A) Mean dopamine concentration change following temporally isolated Omission Probes (latency $>5 \mathrm{~s}$ since previous stimulation) and corresponding time points from the Passive Playback phase $(n=8$ mice; permutation test, $P=0.0005)$. (B) Mean change in dopamine concentration following temporally isolated Omission Probes and equivalent time points from Playback phase (paired $t$ test, $\left.t_{7}=3.511, P=0.0098\right)$. (C) Mean dopamine concentration changes evoked by SelfStimulations without (left) or with (right) an additional lever press during the ongoing stimulation, and the corresponding Passive Playback stimulations ( $n=9$ mice; permutation tests: SelfStimulation with no press during stim vs. its Playback, $P=0.0011$; Self-Stimulation with press during stim vs. its Playback, $P=0.0003$; Self-Stimulation with vs. no press during stim, $P=$ 0.0028). (D) Mean change in dopamine concentration for Self-Stimulations with or without additional presses during the stimulation, and their Playback (two-way repeated-measures ANOVA: main effect of Press, $F_{1,8}=8.144, P=0.0214$; main effect of Session Phase, $F_{1,8}=$ 16.62, $P=0.0035$; Sidak's multiple comparisons tests: Self-Stimulation with vs. no additional press, $P=0.0350$; Self-Stimulation without additional press vs. Playback, $P=0.0163$; SelfStimulation with additional press vs. Playback, $P=0.0011)$. (E) Mean dopamine concentration changes during Omission Probes with or without additional presses during the probe timeout period ( $n=8$ mice; permutation tests: Omission Probe with no timeout press vs. 0, magenta bar, $P=0.002$; Omission Probe with timeout press vs. 0, blue bar, $P=0.0001$; Omission Probe with vs. without press, black bar, $P=0.0003$ ). (F) Mean change in dopamine concentration at early $(1 \mathrm{~s})$ vs. late $(5 \mathrm{~s})$ time points during Omission Probes with or without additional presses during the timeout period (two-way repeated-measures ANOVA: main effect of Press, $F_{1,7}=8.181, P=$ 0.0243; Press by Time interaction, $F_{1,7}=16.70, P=0.0047$; Sidak's multiple comparisons tests: with vs. no press, late, $P=0.0025$; no press, early vs. late, $P=0.0444$; with press, early vs. late, $P=0.0479$ ). (G) Schematic of experimental preparation for in vivo extracellular electrophysiology recordings with optogenetic identification of SNc dopamine neurons. $(\mathbf{H})$ Waveforms of optogenetically identified dopamine neuron for spontaneous (top) and laserevoked (bottom) spikes (Pearson correlation, $r=0.9983, P<0.0001$ ). (I) Raster plot (top) and peri-event time histogram (bottom) of dopamine neuron response to 10-ms optogenetic stimulation pulse. Each row in the raster represents one stimulation, and black ticks are spikes. (J) Raster plot of the same dopamine neuron responses aligned to Self-Stimulation (top) and Passive Playback stimulations (bottom). (K) Firing rate of the optogenetically identified dopamine neuron in $(\mathrm{J})$ in response to Self-Stimulation versus Passive Playback stimulations. (L) Difference trace for the dopamine neuron in $(\mathrm{J})$ depicting Self-Stimulation minus Playback difference in stimulation-evoked firing rate between session phases. OPr, Omission Probe; PrP, Probe Playback. 
A

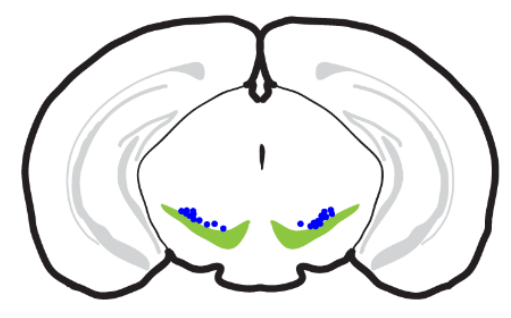

C

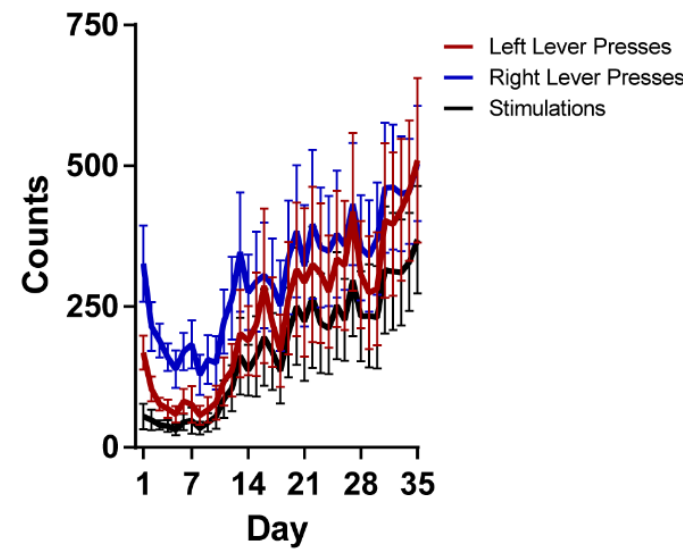

$E$

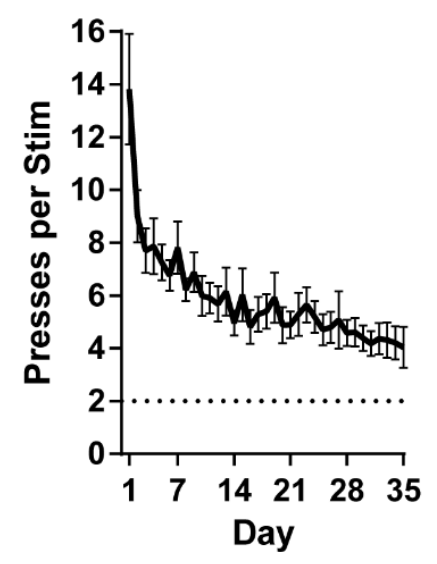

$\mathrm{H}$

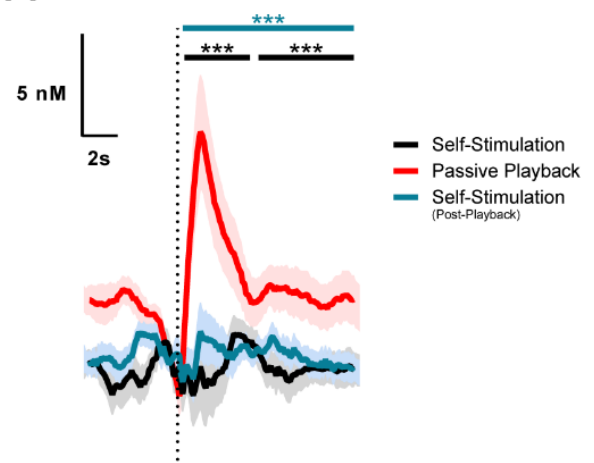

B

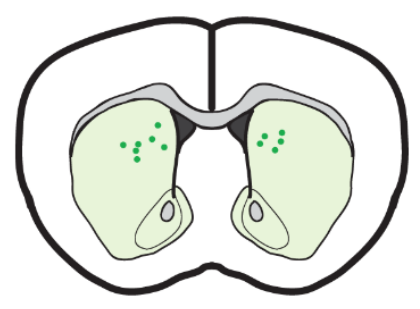

D

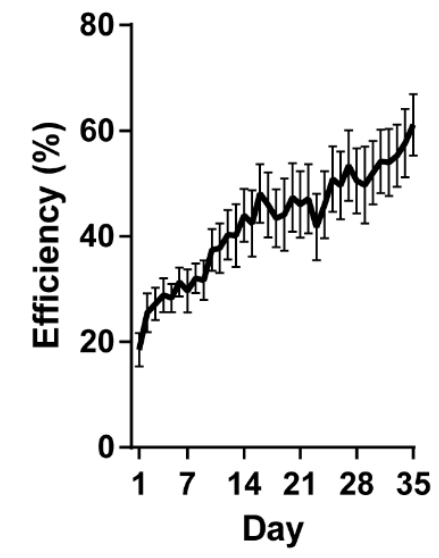

F
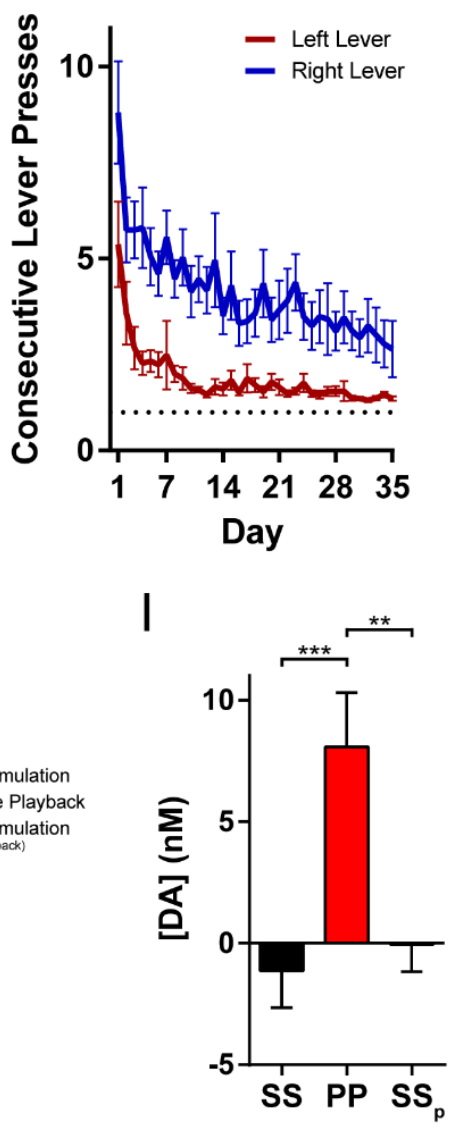
Figure 3-figure supplement 1. Left-Right Sequence Cohort Histology, Additional Behavior, and Session Phase Order Control. (A) Fiber optic placement for mice in the LR sequence cohort. (B) FSCV electrode placement for mice in the LR sequence cohort. (C) Presses on each lever and stimulations earned across days of training $(n=13$ mice; statistics for stimulations presented in Fig. 3B; two-way repeated-measures ANOVA for Lever by Day: main effect of Day, $F_{34,408}=3.189, P<0.0001$; main effect of Lever, $F_{1,12}=16.67, P=0.0015$; Lever by Day interaction, $F_{34,408}=1.535, P=0.0307$ ). (D) Efficiency across days of training, calculated as the number of stimulations per pair of lever presses (one-way repeated-measures ANOVA, $\left.F_{34,408}=6.936, P<0.0001\right)$. (E) Total presses per stimulation (either lever) across days of training (one-way repeated-measures ANOVA, $\left.F_{34,408}=8.122, P<0.0001\right)$. $(\mathbf{F})$ Consecutive presses on each lever across days of training (two-way repeated-measures ANOVA: main effect of Day, $F_{34,408}=8.430, P<0.0001$; main effect of Lever, $\left.F_{1,12}=23.07, P=0.0004\right)$. (G) Contingency degradation test: $30 \mathrm{~min}$ of LR sequence opto-ICSS followed by $30 \mathrm{~min}$ contingency degradation test phase $\left(\mathrm{n}=10\right.$ mice; paired $\mathrm{t}$ test, $\left.t_{9}=3.458, P=0.0072\right) .(\mathrm{H})$ Mean dopamine concentration change evoked by LR Self-Stimulation before or after the Passive Playback phase. The pre-playback Self-Stimulation (black) and Passive Playback responses are the same data as Fig. $3 \mathrm{H}$, and the post-playback Self-Stimulation (teal) is an additional 30 min phase of LR sequence opto-ICSS following the Playback phase to control for potential order effects $(n=12$ mice; permutation tests: $P S=0.0001$ for all time clusters, black bars for pre-playback Self-Stimulation vs. Playback, teal bar for post-playback Self-Stimulation vs. Playback). (I) Mean change in dopamine concentration for Self-Stimulation before or after Passive Playback (one-way repeated-measures ANOVA, $F_{2,22}=26.39, P<0.0001$; Tukey's multiple comparisons tests: Self-Stimulation (pre) vs. Playback, $P<0.0001$; Self-Stimulation (post) vs. Playback, $P<0.0001$ ). SS, Self-Stimulation; CD, Contingency Degradation; PP, Passive Playback; $\mathrm{SS}_{\mathrm{P}}$, Self-Stimulation (post-Playback). 